\section{Fatty acid-binding proteins and fatty acid synthase influence glial reactivity and promote the formation of Müller glia-derived progenitor cells in the avian retina}

1 Department of Neuroscience, College of Medicine, The Ohio State University, Columbus, $\mathrm{OH}$

2 Solomon H. Snyder Department of Neuroscience, Johns Hopkins University School of Medicine, Baltimore, MD.

*corresponding author: Andy J. Fischer, Department of Neuroscience, Ohio State University, College of Medicine, 3020 Graves Hall, 333 W. $10^{\text {th }}$ Ave, Columbus, $\mathrm{OH}$ 43210-1239, USA. Telephone: (614) 292-3524; Fax: (614) 688-8742; email: Andrew.Fischer@osumc.edu

Abbreviated title: FABPs and FASN in Müller glia and Müller glia-derived progenitor cells

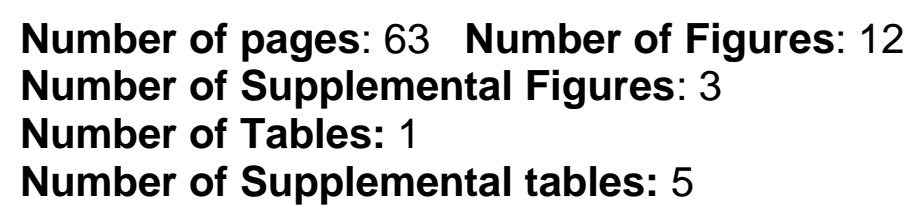

Author Contributions: WAC designed and executed experiments, gathered data, constructed figures and contributed to writing the manuscript. AT and $\mathrm{MH}$ executed experiments, and gathered data. HE coordinated experiments, performed bioinformatic analyses and contributed to writing the manuscript. TH and SB established some of the scRNA-seq libraries. AJF designed experiments, analyzed data, constructed figures and contributed to writing the manuscript.

Acknowledgements: This work was supported by RO1 EY032141- 01 (AJF) and UO1 EY027267-04 (AJF, SB). 


\section{Abstract}

The capacity for retinal regeneration varies greatly across vertebrates species. A recent comparative epigenetic and transcriptomic investigation of Müller glial (MG) in the retinas of fish, birds and mice revealed that Fatty Acid Binding Proteins (FABPs) are among the most highly up-regulated genes in activated chick MG (Hoang et al., 2020). Herein we provide an in-depth follow-up investigation to describe patterns of expression and how FABPs and fatty acid synthase (FASN) influence glial cells in the chick retina. During development, $F A B P 7$ is highly expressed by embryonic retinal progenitor cells (eRPCs) and maturing MG, whereas FABP5 is gradually up-regulated in maturing MG and remains elevated in mature glial cells. PMP2 (FABP8) is expressed by oligodendrocytes and FABP5 is expressed by non-astrocytic inner retinal glial cells, and both of these FABPs are significantly up-regulated in activated MG in damaged or growth factor-treated retinas. In addition to suppressing the formation of MGPCs, we find that FABP-inhibition suppressed the accumulation of proliferating microglia, although the microglia appeared highly reactive. ScRNA-seq analyses of cells treated with FABP-inhibitor revealed distinct changes in patterns of expression suggesting that FABPs are involved in the transitions of MG from a resting state to a reactive state and conversion from reactive MG to MGPCs. Inhibition of FABPs in undamaged retinas had a significant impact upon the transcriptomic profiles of MG, with up-regulation of genes associated with gliogenesis, decreases in genes associated with neurogenesis, and suppression of the ability of MG to become MGPCs. scRNA-seq analyses of microglia indicated that FABP inhibition enhances gene modules related to reactivity, proliferation and cytokine signaling. We find that the proliferation of retinal progenitors in the circumferential marginal zone $(C M Z)$ is unaffected by FABP-inhibitor. Upstream of FABP activity, we inhibited FASN in damaged retinas, which reduced numbers of dying cells, increased the proliferation of microglia, and potently suppressed the formation MGPCs in damaged retinas. We conclude that the activity of FASN and FABPs are required early during the formation of proliferating MGPCs. Fatty acid metabolism and cell signaling involving fatty acids are

69 important in regulating glial homeostasis in the retina, and the dedifferentiation and proliferation of microglia and MGPCs. 


\section{Introduction}

The process of retinal regeneration varies greatly across vertebrate species. In the fish, retinal neuronal regeneration is a robust process that restores functional cells and visual acuity following injury, whereas this process is far less robust in birds and absent in mammals (Hitchcock and Raymond, 1992; Karl et al., 2008; Raymond, 1991). Müller glia (MG) have been identified as the cell of origin for progenitors in regenerating retinas (Bernardos et al., 2007; Fausett and Goldman, 2006; Fausett et al., 2008; Fischer and Reh, 2001; Ooto et al., 2004). In normal healthy retinas, MG are the predominant type of support cell that provide structural, metabolic, visual cycle, and synaptic support (Reichenbach and Bringmann, 2013). In response to damage, certain growth factors or drug treatments, MG can be stimulated to become reactive, dedifferentiate, up-regulate progenitor-related genes, re-enter the cell cycle and produce progeny that differentiate as neurons (Fischer and Bongini, 2010; Gallina et al., 2014a; Wan and Goldman, 2016).

In mammalian retinas, significant stimulation such as forced expression of $A s c / 1$, inhibition of histone deacetylases and neuronal damage is required to reprogram MG into progenitor-like cells that produce a few new neurons (Jorstad et al., 2017; Pollak et al., 2013; Ueki et al., 2015). Alternatively, deletion of Nfia, Nfib and Nfix in mature MG combined with retinal damage and treatment with insulin+FGF2 results in reprogramming of MG into cells that resemble inner retinal neurons (Hoang et al., 2020). Blockade of Hippo-signaling via forced expression of degradation-resistant YAP1 drives the proliferation of mature MG in the mouse retina (Hamon et al., 2019; Rueda et al., 2019), but it remains unknown whether any of the progeny differentiate as neurons. In addition, viral delivery of reporters, $\beta$-catenin, Otx2, Crx and Nrl may reprogram MG into photoreceptors (Yao et al., 2018), but there are concerns that the viral vectors and

97 mini-promoters used in these studies are prone to leaky expression in neurons

98 (Blackshaw and Sanes, 2021). In the chick retina, MG readily reprogram into progenitor-

99 like cells that proliferate, but the progeny have a limited capacity to differentiate as

100 neurons (Fischer and Reh, 2001; Fischer and Reh, 2003). Understanding the 
102 progeny is important to harnessing the regenerative potential of MG in warm-blooded 103 vertebrates.

$104 \quad$ Fatty acid synthesis, metabolism and signaling are likely to be key components 105 of regulating neuronal progenitor cells. Fatty Acid Binding Proteins (FABPs) are 106 cytosolic lipid-binding proteins, that mediate fatty acid metabolism and cell-signaling, 107 and have highly conserved primary and tertiary structures across species from 108 Drosophila to humans (Hanhoff et al., 2002; Smathers and Petersen, 2011). FABPs are 109 known to bind to poly-unsaturated fatty acids (PUFAs) including arachidonic acid and docosahexaenoic acid and have been shown to regulate signal transduction, neurotransmission, proliferation, differentiation, and cell migration (Allen et al., 2007;

112 Dawson and Xia, 2012; Tripathi et al., 2017; Yamashima, 2012). Very little is known about the cellular mechanisms and patterns of expression of FABPs in the retina. In mammals, FABP3, 5, and 7 have been identified in the brain, retina, and radial glia with demonstrated roles in differentiation and cell fate determination (Owada, 2008; Sellner, 1993; Sellner et al., 1995) llen et al., 2007; Dawson and Xia, 2012; Tripathi et al., 2017; Yamashima, 2012). Further evidence indicates that FABPs in the CNS modulate endocannabinoid, Peroxisome Proliferator-Activated Receptor (PPAR), NF-kB, and CREB signaling (Bogdan et al., 2018; Peng et al., 2017; Tripathi et al., 2017; Yamashima, 2012). NF-kB has been implicated as a key signaling "hub" that suppresses the formation of MGPCs in chicks and mice, but not zebrafish (Hoang et al., 2020; Palazzo et al., 2020).

We have previously reported that FABP5 and PMP2 are highly up-regulated in MG in NMDA-damaged retinas, and that inhibition of FABPs potently suppresses the formation of proliferating MGPCs (Hoang et al., 2020). However, few details are known about the mechanisms by which FABPs act to influence the formation of MGPCs, coordinate with other cell-signaling pathways, influence the reactivity of microglia, and induce changes in gene expression following FABP-inhibition. Accordingly, this study investigates how FABPs and Fatty Acid Synthase (FASN) influence reprogramming of MG into MGPCs and analyze transcriptomic changes downstream of FABP inhibition in damaged and growth factor-treated retinas in the chick model system. 


\section{Methods and Materials:}

Animals:

The animals approved for use in these experiments was in accordance with the guidelines established by the National Institutes of Health and IACUC at The Ohio State University. Newly hatched P0 wildtype leghorn chicks (Gallus gallus domesticus) were obtained from Meyer Hatchery (Polk, Ohio). Post-hatch chicks were maintained in a regular diurnal cycle of 12 hours light, 12 hours dark (8:00 AM-8:00 PM). Chicks were housed in stainless-steel brooders at $25^{\circ} \mathrm{C}$ and received water and Purinatm chick starter ad libitum.

Fertilized eggs were obtained from the Michigan State University, Department of Animal Science. Eggs were incubated at a constant $37.5^{\circ} \mathrm{C}$, with a $1 \mathrm{hr}$ period at room temperature with a cool-down period every $24 \mathrm{hrs}$. The eggs were rocked every 45 minutes and held at a constant relative humidity of $45 \%$. Embryos were harvested at various time points after incubation and staged according to guidelines established by

Intraocular injections:

Chicks were anesthetized with $2.5 \%$ isoflurane mixed with oxygen from a nonrebreathing vaporizer. The technical procedures for intraocular injections were performed as previously described (Fischer et al., 1998). With all injection paradigms, both pharmacological and vehicle treatments were administered to the right and left eye respectively. Compounds were injected in $20 \mu$ sterile saline with $0.05 \mathrm{mg} / \mathrm{ml}$ bovine serum albumin added as a carrier. Compounds included: NMDA (38.5nmol or 154 $\mu$ g/dose; Millipore Sigma), FGF2 (250 ng/dose; R\&D systems), BMS309403 (Millipore Sigma), C75 (Millipore Sigma), G28UCM (Millipore Sigma). 5-Ethynyl-2'-deoxyuridine (EdU, ThermoFischer) was injected into the vitreous chamber to label proliferating cells. Injection paradigms are included in each figure.

\section{Single Cell RNA sequencing of retinas}


Retinas were obtained from embryonic and hatched chicks. Retinas were dissociated in a $0.25 \%$ papain solution in Hank's balanced salt solution (HBSS), $\mathrm{pH}=$ 7.4, for 30 minutes, and suspensions were frequently triturated. The dissociated cells were passed through a sterile $70 \mu \mathrm{m}$ filter to remove large particulate debris. Dissociated cells were assessed for viability (Countess II; Invitrogen) and cell-density diluted to 700 cell/ $\mu \mathrm{l}$. Each single cell cDNA library was prepared for a target of 10,000 cells per sample. The cell suspension and Chromium Single Cell 3' V2 or V3 reagents (10X Genomics) were loaded onto chips to capture individual cells with individual gel beads in emulsion (GEMs) using the 10X Chromium Cell Controller. cDNA and library amplification and for an optimal signal was 12 and 10 cycles respectively. Sequencing was conducted on Illumina HiSeq2500 (Genomics Resource Core Facility, John's Hopkins University), or Novaseq6000 (Novogene) using 150 paired-end reads. Fasta sequence files were de-multiplexed, aligned, and annotated using the chick ENSMBL database (GRCg6a, Ensembl release 94) by using 10X Cell Ranger software. Gene expression was counted using unique molecular identifier bar codes and gene-cell matrices were constructed. Using Seurat toolkits Uniform Manifold Approximation and Projection for Dimension Reduction (UMAP) plots were generated from aggregates of multiple scRNA-seq libraries (Butler et al., 2018; Satija et al., 2015). Seurat was used to construct gene lists for differentially expressed genes (DEGs), violin/scatter plots and dot plots. Significance of difference in violin/scatter plots was determined using a Wilcoxon Rank Sum test with Bonferroni correction. Monocle was used to construct unguided pseudotime trajectories and scatter plotters for MG and MGPCs across pseudotime (Qiu et al., 2017a; Qiu et al., 2017b; Trapnell et al., 2012). cells within scRNA-seq datasets (Cabello-Aguilar et al., 2020). Genes that were used to identify different types of retinal cells included the following: (1) Müller glia: GLUL, VIM, SCL1A3, RLBP1, (2) MGPCs: PCNA, CDK1, TOP2A, ASCL1, (3) microglia: C1QA, C1QB, CCL4, CSF1R, TMEM22, (4) ganglion cells: THY1, POU4F2, RBPMS2, NEFL, NEFM, (5) amacrine cells: GAD67, CALB2, TFAP2A, (6) horizontal cells: PROX1, CALB2, NTRK1, (7) bipolar cells: VSX1, OTX2, GRIK1, GABRA1, and (7) cone photoreceptors: CALB1, GNAT2, OPN1LW, and (8) rod photoreceptors: RHO, NR2E3, 
ARR1. Gene Ontology (GO) enrichment analysis was performed using ShinyGO V0.65 (http://bioinformatics.sdstate.edu/go/). scRNA-seq libraries can be queried at https://proteinpaint.stjude.org/F/2019.retina.scRNA.html or gene-cell matricies downloaded from GitHub at https://github.com/fischerlab3140/scRNAseq libraries

Fixation, sectioning and immunocytochemistry:

Retinal tissue samples were formaldehyde fixed, sectioned, and labeled via immunohistochemistry as described previously (Fischer et al., 2006; Ghai et al., 2009). Antibody dilutions and commercial sources for images used in this study are described in table 1. Observed labeling was not due to off-target labeling of secondary antibodies or tissue auto-fluorescence because sections incubated exclusively with secondary antibodies were devoid of fluorescence. Secondary antibodies utilized include donkeyanti-goat-Alexa488/568, goat-anti-rabbit-Alexa488/568/647, goat-anti-mouseAlexa488/568/647, goat-anti-rat-Alexa488 (Life Technologies) diluted to 1:1000 in PBS and $0.2 \%$ Triton $\mathrm{X}-100$.

Labeling for EdU:

For the detection of nuclei that incorporated EdU, immunolabeled sections were

212 fixed in $4 \%$ formaldehyde in $0.1 \mathrm{M}$ PBS pH 7.4 for 5 minutes at room temperature.

213 Samples were washed for 5 minutes with PBS, permeabilized with $0.5 \%$ Triton X-100 in 214 PBS for 1 minute at room temperature and washed twice for 5 minutes in PBS. Sections were incubated for 30 minutes at room temperature in a buffer consisting of $100 \mathrm{mM}$

216 Tris, $8 \mathrm{mM} \mathrm{CuSO}_{4}$, and $100 \mathrm{mM}$ ascorbic acid in $\mathrm{dH}_{2} \mathrm{O}$. The Alexa Fluor 568 Azide

217 (Thermo Fisher Scientific) was added to the buffer at a 1:100 dilution.

Terminal deoxynucleotidyl transferase dUTP nick end labeling (TUNEL):

The TUNEL assay was implemented to identify dying cells by imaging

221 fluorescent labeling of double stranded DNA breaks in nuclei. The In Situ Cell Death Kit

222 (TMR red; Roche Applied Science) was applied to fixed retinal sections as per the 223 manufacturer's instructions. 
Photography, measurements, cell counts and statistics:

Microscopy images of retinal sections were captured with the Leica DM5000B microscope with epifluorescence and the Leica DC500 digital camera. High resolution confocal images were obtained with a Leica SP8 available in The Department of Neuroscience Imaging Facility at The Ohio State University. Representative images are modified to have enhanced color, brightness, and contrast for improved clarity using Adobe Photoshop. In EdU proliferation assays, a fixed region of retina was counted and average numbers of Sox2 and EdU co-labeled cells. The retinal region selected for investigation was standardized between treatment and control groups to reduce variability and improve reproducibility.

Similar to previous reports (Ghai et al., 2009), immunofluorescence intensity was quantified by using Image $\mathrm{J}(\mathrm{NIH})$. Identical illumination, microscope, and camera settings were used to obtain images for quantification. Retinal areas were sampled from digital images. These areas were randomly sampled over the inner nuclear layer (INL). MS Excel and GraphPad Prism 6 were used for statistical analyses. Measurement for immunofluorescence in the nuclei of MG/MGPCs were made by from single optical confocal sections by selecting the total area of pixel values above threshold $(\geq 70)$ for

242 Sox2 or Sox9 immunofluorescence (in the red channel) and copying nuclear labeling from only MG (in the green channel). Measurements of pS6 immunofluorescence were

244 made for a fixed, cropped area $\left(14,000 \mu \mathrm{m}^{2}\right)$ of INL, OPL and ONL. Measurements 245 were made for regions containing pixels with intensity values of 70 or greater $(0=$ black and 255 = saturated). The total area was calculated for regions with pixel intensities above threshold. The intensity sum was calculated as the total of pixel values for all pixels within threshold regions.

For statistical evaluation of differences in treatments, a two-tailed paired $t$-test was applied for intra-individual variability where each biological sample also served as its own control. For two treatment groups comparing inter-individual variability, a twotailed unpaired $t$-test was applied. For multivariate analysis, an ANOVA with the associated Tukey Test was used to evaluate any significant differences between

254 multiple groups. 
Results:

\section{Expression of $F A B P s$ during embryonic retinal development:}

scRNA-seq libraries were established for retinal cells at embryonic day 5 (E5), E8, E12, and E15. These libraries yielded 22,698 cells after filtering to exclude doublets, cells with low UMI/genes per cell, and high mitochondrial gene-content. UMAP plots of aggregate libraries formed clusters of retinal cells that correlated to developmental stage and cell type (Fig. 1a). Types of cells were identified based on expression of wellestablished markers. Retinal progenitor cells (RPCs) from E5 and E8 retinas were identified by expression of $A S C L 1, C D K 1$, and TOP2A. (Fig. 1b). Maturing MG were identified by expression of GLUL, RLBP1 and SLC1A3 (Fig. 1b). FABP5 and FABP7 were expressed by different types of developing retinal cells at different stages of development, whereas PMP2 (FABP8) was not widely expressed by embryonic retinal cells (Fig. 1c). FABP5 was predominantly expressed by maturing bipolar and amacrine cells (Figs. 1c,d). By comparison, FAPB7 was predominantly expressed by early retinal progenitor cells (eRPCs) from E5 and E8 retinas and at elevated levels in immature MG at E8, whereas levels decreased in maturing MG at E12 and E15 (Figs.1c,d,f). FABP7 was also expressed by maturing bipolar and amacrine cells from E8 retinas (Figs. 1c,d).

To further assess patterns of expression we re-embedding of eRPCs and MG to

274 order cells across pseudotime in an unsupervised manner. Pseudotime-ordering

275 revealed a continuum of cells with early eRPCs and maturing MG at opposite ends of 276 the trajectory (Fig. 1e). Across pseudotime levels of GLUL increased, while levels of $277 C D K 1$ decreased (Fig. 1e,f). Like GLUL, the expression of FABP5 increases from retinal 278 progenitors to maturing MG (Fig. 1e-g). By contrast, levels of $F A B P 7$ were relatively 279 high in eRPCs, increased in differentiating immature MG, and decreased in MG during 280 later stages of development (Figs. 1e-g). Immunolabeling for PMP2 confirmed findings 281 from scRNA-seq. PMP2 was not expressed in the developing retina until E16, when 282 PMP2-immunofluorescence was detected near the vitread surface of the retina in cells 283 that resembled oligodendrocytes (Fig. 1h). These findings indicate that FABP5 and $284 F A B P 7$ are dynamically and highly expressed in eRPCs and MG through the course of 285 embryonic development. 


\section{Expression of FABPs in damage to the chick retina:}

We sought to provide a detailed description of patterns of expression of FABPs in the retinas of normal and hatched chick by using scRNA-seq. Libraries were aggregated for retinal cells obtained from control and NMDA-damaged retinas at different time points (24, 48 and $72 \mathrm{hrs}$ ) after treatment for a total of 57,230 cells (Fig. 2a). We have previously used these chick scRNA-seq databases to compare MG and MGPCs across fish, chick and mouse model systems (Hoang et al., 2020) and characterize expression patterns of genes related to NFkB-signaling (Palazzo et al., 2020), midkine-signaling (Campbell et al., 2021), matrix metalloproteases (Campbell et al., 2019), and endocannabinoid-signaling (Campbell et al., 2021b). UMAP-clustered cells were identified based on well-established patterns of expression (Fig. 2a,b). Resting MG formed a discrete cluster of cells and expressed high levels of GLUL, RLBP1 and SLC1A3 (Fig. 2a,b). After damage, MG down-regulate markers of mature glia as they transition into reactive glial cells and into progenitor-like cells that up-regulate TOP2A, CDK1 and ESPL1 (Fig. 2a,b). FABP5 and PMP2 were expressed at low levels in relatively few resting $M G$ in undamaged retina, whereas $F A B P 7$ was widely expressed by the majority of resting $M G$ (Fig. 2c,d). FABP7 and $P M P 2$ were detected in oligodendrocytes and Non-astrocytic Inner Retinal Glia (NIRGs). NIRG cells are a distinct type of glial cell that has been described in the retinas of birds (Rompani and Cepko 2010; Fischer et al., 2010) and some types of reptiles (Todd et al., 2016b). Following NMDA-induced retinal damage, levels of FABP5, FABP7 and PMP2 were significantly increased in activated MG at 24 hrs (Fig. 2c,d). Levels of FABP5 and PMP2 were significantly reduced in activated MG at $48 \mathrm{hrs}$ and $72 \mathrm{hrs}$, but remained elevated in proliferating MGPCs (Fig. 2c,d).

We re-ordered $M G$ along pseudotime trajectories to better assess changes in expression of $F A B P s$ during the transition of MG to progenitor cells. We identified 5

313 pseudotime states and a trajectory that included distinct branches for resting Müller glia

314 (state 1), activated Müller glia (state 5), transitional Müller glia (states 2,3), and MGPCs

315 (state 4) (Fig. 2e). Dimensional reduction to a single pseudotime axis placed resting 316 Müller glia (high levels of GLUL) to the left and highly activated MG and MGPCs (high

317 levels of $C D K 1$ ) the far right of the pseudotime axis (Fig. 2e-f). The expression of 
FABP5 and PMP2 across pseudotime positively correlated with a transition toward an MGPC-phenotype, and inversely correlated to resting glial phenotypes (Fig. 2f,g). Levels of $F A B P 7$ were not significantly different across most pseudotime states, with the exception of elevated levels in state 4 which is occupied by MGPCs, compared to levels in resting and activated MG (Fig. 2f,g).

We next assessed patterns of expression of FABPs in scRNA-seq libraries from time-points soon after NMDA at 3 and 12 hrs after treatment. These libraries were generated with newer, more sensitive reagents and did not integrate well with older libraries generated with less sensitive reagents. UMAP ordering of MG revealed distinct clusters of cells which closely match treatment groups and included resting MG, early activated MG, 3 groups of activated MG and 2 groups of MGPCs (supplemental Fig. 1a,b). Levels of FABP5 were reduced, but expressed by a larger percentage of MG at 3hrs after NMDA, levels increased in activated MG, and further increased in MGPCs (supplemental Fig. 1e,h). FABP7 was down-regulated in MG at 12hrs, but increased in MGPCs at 48hrs after NMDA (supplemental Fig. 1f,h). Levels of PMP2 were not increased in MG until 12hrs after NMDA-treatment, and remained elevated in MGPCs through 48hrs after treatment (supplemental Fig. 1g,h).

Immunolabeling for PMP2

We next sought to characterize PMP2 immunolabeling in normal retinas. We found that PMP2 immunofluorescence was observed in NIRG cells, but only in peripheral regions of retina (Fig. 3a). Although the NIRG cells were often observed in the proximal INL and had morphologies reminiscent of amacrine cells; these cells were negative for amacrine cell markers including AP2 $\alpha$, Islet1 and tyrosine hydroxylase (Fig. 3a). PMP2-immunolabeling was prevalent in oligodendrocytes extended processes into the NFL, and were negative for Glutamine Synthase (GS; Fig. 3b). The PMP2-positive

344 oligodendrocytes where immunoreactive for Olig2 (Fig. 3c), Sox10 (Fig. 3g) and HuC/D

345 (Fig. 3d). HuC/D is thought to be a neuron-specific marker, but appears to be up346 regulated in PMP2+ oligodendrocytes after NMDA-treatment (Fig. 3d) and a few of

347 these cells proliferate (accumulate EdU) in central and peripheral regions of the 
after NMDA-treatment. Further, we failed to observe PMP2 in NIRG cells (Sox2+/Nkx2.2+ cells in the IPL) in central regions of control or NMDA-damaged retinas (Fig. 3h,i). Collectively, these patterns of immunolabeling are consistent with scRNAseq data for oligodendrocytes and NIRG cells and patterns of expression for PMP2, SOX10, OILG2 and ELAVL4 (HuD; Fig. 3j). Further, NIRG cells expressed FABP5 and FABP7, whereas oligodendrocytes expressed PMP2 (Fig. 3j).

\section{FABPs in eRPC, MGPCs and CMZ progenitors}

We next sought to directly compare levels of FABPs between different types of retinal progenitor cells. We aggregated progenitors from E5 and E8 retinas, and MGPCs from retinas treated with NMDA and/or insulin and FGF2 (Fig. 4a). UMAP-ordering of cells revealed 2 distinct clusters of cells for eRPCs and MGPCs (Fig. 4b), with both clusters expressing high levels of proliferation-associated genes including PCNA, SPC25, TOP2A and CDK1 (Fig. 4b,c). MGPCs express high levels of FABP5 and PMP2 with significantly higher levels in MGPCs from retinas treated with insulin and FGF2, whereas eRPCs did not express FABP5 or PMP2 (Fig. 4c-f). By contrast, levels of $F A B P 7$ were higher in proliferating eRPCs than in proliferating MGPCs with significantly lower levels in MGPCs from retinas treated with insulin and FGF2 (Fig. 4f).

We next examined whether retinal progenitors in the circumferential marginal zone (CMZ) expressed PMP2. Proliferating retinal progenitors with limited neurogenic potential are known to reside in a CMZ in the post-hatch chick eye (Fischer and Reh, 2000). Since our scRNA-seq databases did not include CMZ progenitors at the far peripheral edge of the retina, we immunolabeled sections of $\mathrm{CMZ}$ with antibodies to PMP2. PMP2-immunolabeling was present at relatively low levels in MG and CMZ progenitors at the far peripheral edge of the retina (Fig. 4h). Levels of PMP2-

374 immunoreactivity appeared elevated in MG and CMZ progenitors in retinas treated with

375 insulin and FGF2 (Fig. 4h). Although, the proliferation of CMZ progenitors was increased by injections of insulin and FGF2, the proliferation of CMZ progenitors was unaffected by FABP inhibitor (Fig. 4i-k). 
FABPs in retinas treated with insulin and FGF2

We next examined FABP expression in MG and MGPCs in the absence of neuronal damage. In the chick retina the formation of proliferating MGPCs can be induced by consecutive daily injections of Fibroblast growth factor 2 (FGF2) and insulin in the absence of neuronal damage (Fischer et al., 2002; Fischer et al., 2009b; Fischer et al., 2014b; Ritchey et al., 2012). Eyes were treated with two or three consecutive daily doses of FGF2 and insulin, and retinas were processed to generate scRNA-seq libraries. UMAP ordering of cells revealed distinct clusters that were segregated based on cell type (Fig. 5a). MG glia were identified based on the expression of VIM, GLUL and SLC1A3, and MGPCs were identified based on expression of TOP2A, NESTIN, CCNB2 and CDK1 (Fig. 5b). Resting MG from saline-treated retinas formed a cluster of cells distinct from MG from retinas treated with either two or three doses of FGF2+insulin (Fig. 5b). Additionally, MG treated with 2 versus 3 doses of insulin and FGF2 were sufficiently dissimilar to follow different pseudotime trajectories, with MGPCs from retinas treated with 3 doses of insulin+FGF2 occupying a distinct branch (Fig. 5e). Similar to patterns of expression seen in NMDA-damaged retinas, FABP5, FABP7 and $P M P 2$ were significantly increased in MG treated with insulin and FGF2 (Fig. 5d). Reduction of pseudotime trajectories to one axis revealed increases in levels of FABP5 and $P M P 2$ across pseudotime that paralleled increasing numbers of proliferating MGPCs that expressed $C D K 1$, whereas levels of $F A B P 7$ were relatively unchanged across pseudotime (Fig. 5f).

We next compared levels of FABPs in MG and MGPCs in retinas treated with insulin+FGF2 \pm NMDA. We probed a large aggregate scRNA-seq library of more than 55,000 cells wherein UMAP ordering revealed discrete clustering of resting MG, activated MG and MGPCs (supplemental Fig. 2a). Levels of FABP5 and PMP2 were lowest in resting MG, and highest in activated MG at 24hrs after NMDA and MGPCs compared to the elevated levels seen in MG treated with insulin+FGF2,

407 NMDA+insulin+FGF2 and activated MG at 48+72 hrs after NMDA (supplemental Fig. 2f-

408 h). Patterns of expression for FABP7 were similar to those seen for FABP5 and PMP2, 409 although higher levels of expression were observed in both reactive and, in particular, 410 resting MG (supplemental Fig. 2f-h). By comparison, levels of $F A S N$ were highest in 
411 resting MG, lowest in MGPCs, and at intermediate levels in activated MG observed

412 following injury or growth factor treatment (supplemental Fig. 2f-h).

To test whether PMP2 was increased when MG were treated with FGF2 we

414 immunolabeled retinal sections. We found that PMP2-immunoreactivity was increased

415 in FGF2-treated MG (Fig. 6a). This effect was not evident in central regions of the 416 retina, but was apparent in peripheral regions (Fig. 6a). Some of the PMP2+/Sox2+ MG

417 were labeled for EdU (Fig. 6b), indicating that these cells were proliferating MGPCs. We 418 next investigated whether FABP-inhibition influenced the formation of MGPCs in the 419 absence of neuronal damage. We have reported previously that proliferating MGPCs 420 are formed in peripheral regions of retinas treated with consecutive daily injections of 421 FGF2 alone (Fischer et al., 2014). Accordingly, we applied BMS309403 with FGF2 and 422 probed for the formation of proliferating MGPCs. Inhibition of FABPs significantly 423 reduced numbers of Sox2/EdU-labeled cells in the INL of FGF2-treated retinas (Fig.

$4246 \mathrm{c}, \mathrm{d})$.

Genes regulated by FABP-inhibition

427 To identify transcriptional changes downstream of FABP inhibition we generated 428 scRNA-seq libraries for control retinas and retinas treated with inhibitor (BMS309403) \pm 429 NMDA. Aggregation of the scRNA-seq libraries revealed distinct UMAP clusters of 430 retinal neurons and glia (Fig. 7a,b). UMAP-ordering did not result in distinct clustering of 431 neurons based on treatment, whereas MG were clustered according to treatments (Fig. 432 7a,b). Genes upregulated in activated MG included HBEGF, TUBB6 and TGFB2 (Fig. 433 7d). MGPCs upregulated genes such as CDK1, TOP2A and ESPL1 (Fig. 7e). Resting $434 \mathrm{MG}$ expressed high levels of GLUL, RLBP1 and SLC13A which were downregulated in 435 activated MG (Fig. 7f). We identified differentially expressed genes (DEGs) in MG from 436 retinas treated with BMS, NMDA and BMS+NMDA (Fig. 7c; supplemental tables 1,2,3).

437 BMS treatment of undamaged retinas resulted in significant changes in gene 438 expression, which included down-regulation of 393 genes including markers for resting 439 mature glia such as GLUL, CRABP-I, RLBP1, CA2, SFRP1 and ID4 (Fig. 7g, 440 supplemental table 1). By comparison, BMS-treatment resulted in an up-regulation of 441495 genes including FABPs, secreted factors associated with glial activation and 
442 receptors for TNF-related ligands (Fig. 7g, supplemental table 1). Gene ontology (GO)

443 enrichment analysis of DEGs from saline-BMS-treated MG revealed significant

444 enrichment for up-regulated genes associated with gliogenesis, wound healing and

445 developmental processes (Fig. 7i), whereas down-regulated genes were associated

446 with neurogenesis and cell proliferation (Fig. 7i). BMS-treatment of NMDA-damaged

447 retinas resulted in significant changes in gene expression which included down-

448 regulation of 192 genes including markers for proliferation, pro-glial genes NFIX and

449 ID4, and High Mobility Group (HMG) genes (Fig. 7h, supplemental table 2). By

450 comparison, BMS-treatment resulted in an up-regulation of 114 genes including FABP5

451 and FABP7, secreted factors BMP4, WNT4 and WNT6, and glial reactivity genes such

452 as CD44 (Fig. 7h, supplemental table 2). GO enrichment analysis of down-regulated

453 genes from NMDA/BMS-treated MG revealed significant enrichment for genes

454 associated with proliferation and organelle fission (Fig. 7i). By comparison, GO

455 enrichment analysis for upregulated genes from NMDA/BMS-treated MG revealed

456 significant enrichment for genes associated with growth factor signaling, cell death and

457 cell motility (Fig. 7j).

458 We isolated MG from 4 treatment groups and re-ordered these cells in UMAP

459 plots. The MG formed 5 distinct clusters; 2 clusters of resting MG (occupied

460 predominantly by cells from retinas treated with saline- and BMS-saline), 2 clusters of

461 activated glia (occupied predominantly cells from retinas treated with NMDA, BMS-

462 NMDA and BMS-saline), and MGPCs (occupied predominantly by cells from retinas

463 treated with NMDA alone) (supplemental Fig. 2a-c). This finding suggest that BMS-

464 treatment induces MG to acquire a reactive phenotype. Markers for mature resting MG

465 were significantly down-regulated in activated MG and MGPCs; these markers included

466 GLUL, RLBP1, CSPG5 and ID4 (supplemental Fig. 2d-h). By contrast, activated MG

467 significantly up-regulated markers associated with reactivity such as HBEGF, TGFB2,

468 BMP4, S100A, SDC4, CD44, FABP5, FABP7 and PMP2 (supplemental Fig. 2d-h). Not

469 surprising, MGPCs had elevated levels of proliferation markers such as CDK1, TOP2A

470 and SPC25 (supplemental Fig. 2d-h). GO enrichment analysis for DEGs between

471 resting MG and activated MG revealed significant enrichment for up-regulated genes

472 associated with cell metabolism, structural cytoskeleton, and organelle organization 
473 (supplemental Fig. 2j). By comparison there was significant enrichment for down-

474 regulated genes associated with transcriptional regulation, cell adhesion, cellular

475 projections, and neuronal development (supplemental Fig. 2j). GO enrichment analysis

476 for DEGs between activated MG and MGPCs revealed significant enrichment for up-

477 regulated genes associated with cell division, and enrichment for down-regulated genes

478 associated with nervous system development and neuronal differentiation

479 (supplemental Fig. 2i).

We next isolated the MG, microglia and NIRG cells, re-embedded these cells in

481 UMAP plots and probed for cell signaling networks and putative ligand-receptor

482 interactions using SingleCellSignalR (Cabello-Aguilar et al., 2020). We chose to focus

483 our analyses on the MG, microglia and NIRG cells because there is significant evidence

484 to indicate autocrine and paracrine signaling among MG, microglia and NIRG cells in

485 the context of glial reactivity and the formation of proliferating MGPCs (Fischer et al.,

486 2014b; Wan et al., 2012; White et al., 2017; Zelinka et al., 2012a). Resting MG included

487 cells for saline and BMS-treatment groups, activated MG included cells mostly from

488 BMS-saline, NMDA and BMS-NMDA treatment groups, and MGPCs were

489 predominantly derived the NMDA-treatment group (Fig. 8a-c). Numbers of LR-

490 interactions (significant upregulation of putative ligand and receptor) between cell types

491 in the different treatment groups varied between 70 and 315 (Fig. 8d-g). We performed

492 analyses on glial cells from each treatment group and compared changes across the

493 most significant LR-interactions. For example, LR-interactions included IGF1R and

494 FGFR1 in activated MG to MGPCs, but not when treated with BMS (Fig. 8f,g). By

495 comparison, BMS-treated MG included LR-interactions with TGFBR2 and interactions

496 involving TNFRSF11B, MDK, PTN and PTPRZ1 (Fig. 8f,g). We compared significant

497 changes in LR-interactions among glial cells and interactions unique to treatment

498 groups for undamaged and damaged retinas. We identified 33 LR-interactions specific

499 to saline-treated glia and 148 LR-interactions specific to BMS-treated glia in undamaged

500 retinas (Fig. 8h,i,I,m,p). Glia in undamaged saline-treated retinas included LR-

501 interactions for FGF9-FGFR3/4, BMP2-ACVR2 and BMP6-BMPR2 (Fig. 8h,i,I,m,p). By

502 comparison, glia in undamaged BMS-treated retinas included LR-interactions

503 associated with activated glial phenotypes such as IL1B-IL1RAP, TGFB1/2-TGFBR2, 

HBEGF-CD9/CD82/ERBB2/EGFR, and JAG1/JAG2/PSEN1-NOTCH2 (Fig. 8h,i,l,m,p p). We identified 40 LR-interactions specific to saline/NMDA-treated glia and 86 LRinteractions specific to BMS/NMDA-treated glia in damaged retinas (Fig. 8j,k,n,o,q). LRinteractions unique to glia in NMDA-damaged retinas included BMP-, MDK-, FGF- and DLL1-Notch1-signaling (Fig. 8j,k,n,o,q). By comparison, LR-interactions unique to BMS/NMDA-damaged retinas included JAG1/2/PSEN1-Notch2, INHBA-ACVR2, TGFB1-ITGB3 and WNT5A-LRP2/FZD4 (Fig. 8 j,k,n,o,q).

\section{Inhibition of FABPs in resting MG}

We next sought to investigate and validate changes in cell signaling in damaged retinas treated with FABP-inhibitor. One day after treatment with NMDA \pm BMS there was a significant increase in numbers of dying TUNEL+ cells (Fig. 9a,b). By contrast, we observed a significant decrease in pS6 in MG in damaged retinas treated with BMS (Fig. 9a,b), consistent with findings from SingleCellSignalR where LR-interactions for FGF1-FGFR1 and MDK-ITGA4 are missing with BMS-treatment (Fig. 8q). MDK and FGF are known to active mTor-signaling and up-regulated pS6 in MG in the chick retina (Campbell et al., 2021a; Zelinka et al., 2016). Consistent with these findings, we observed reduced levels of pStat3 in MG nuclei in damaged retinas treated with BMS (Fig. 9a,b). Stat phosphorylation is known to be down-stream of PDGF-signaling (Li et al., 2012; Popielarczyk et al., 2019) and PDGFA-PDGFRA LR-interactions are missing from damaged retinas treated with BMS (Fig. 8q). Similarly, we find reduced levels of pSMAD1/5/8 in MG nuclei in damaged retinas treated with BMS (Fig. 9a,b). This may result from increased signaling through ACVR2A and TGFB1/ITGB3 which may antagonize BMP/SMAD-signaling (Todd et al., 2017).

scRNA-seq data indicated that BMS-treatment of undamaged retina had a significant impact on the transcriptional profile of MG with profiles resembling dedifferentiation and reactive glia. We sought to verify some of the scRNA-seq data by labeling BMS-treated retinas with antibodies to vimentin or PMP2. We found that BMS-

532 treatment significantly increase immunofluorescence for vimentin and PMP2 in MG (Fig.

533 9c,d). Since, BMS treatment appeared to stimulate MG to become reactive and de- 
535 (Hoang et al., 2020), we tested whether BMS treatment primes MG to become 536 proliferating progenitors in damaged retinas. BMS treatment should have primarily

537 inhibited FABP7 in resting MG and this inhibition was predicted to enhance the ability of

538 MG to become proliferating MGPCs. Contrary to expectations, we found that BMS

539 treatment of retinas before NMDA-induced damage suppressed the formation of

540 proliferating MGPCs (Fig. 9e,f). The proliferation of NIRG cells was not affected by BMS

541 treatment prior to NMDA-induced retinal damage (not shown).

\section{Effects of FABP-inhibition in microglia}

544 Retinal microglia and infiltrating macrophage are known to promote the formation 545 of MGPCs in chick and zebrafish retinas (Fischer et al., 2014b; Palazzo et al., 2020;

546 White et al., 2017) and suppress the neuronal differentiation of reprogrammed MG in 547 mouse retinas (Todd et al., 2020). Accordingly, we investigated whether microglia were

548 influenced by treatment with FABP inhibitor. The application of BMS to NMDA-damaged 549 retinas suppressed the accumulation of microglia and significantly reduced numbers of 550 EdU+/CD45+ cells (Fig. 10a,b). The microglia in BMS-NMDA treated retinas appeared 551 to retain a reactive morphology (Fig. 10a). Thus, it is possible that reduced numbers of 552 proliferating MGPCs resulted, in part, from reduced accumulation of reactive monocytes in BMS-treated retinas.

We isolated and UMAP-embedded microglia from retinas treated with saline,

555 BMS and NMDA. UMAP plots revealed distinct clusters of resting microglia, activated and proliferating cells (Fig. 10c). The resting microglia UMAP clusters were

557 predominantly occupied by microglia from saline-treated retinas, whereas microglia from

558 BMS-saline treated retinas were clustered among activated cells (Fig. 10c,d). BMS-

559 treatment of microglia in undamaged retinas resulted in up-regulation of 545 genes,

560 whereas no genes were significantly down-regulated (Fig. 10e,g; supplemental table 4).

561 BMS-treatment in undamaged retinas stimulated microglia to up-regulate genes

562 associate with proliferation, cell signaling, complement, integrins and glial transcription

563 factors (Fig. 10e; supplemental table 4). NMDA-treatment stimulated microglia to

564 significantly up-regulate nearly 1400 genes including FABP5, FABP7, PMP2 and FASN, 
566 treatment of NMDA-damaged retinas resulting if changes in expression of only 6 genes,

567 and PMP2 was among the few genes upregulated by microglia in BMS/NMDA-damaged 568 retinas (Fig. 10f). GO enrichment analysis of DEGs in microglia from retinas treated with 569 saline \pm BMS indicated groups of genes associated with cellular biogenesis, regulation 570 of cell death and hydrolytic/catabolic processes (Fig. 10h). Thus, it is not surprising that

571 the BMS-treated microglia were embedded among microglia from NMDA-damaged

572 retinas in UMAP plots (Fig. 10c,d). GO enrichment analysis of DEGs in microglia from

573 retinas treated with saline \pm NMDA indicated groups of genes associated with lytic 574 enzyme activity, lysosomal activity and cellular biogenesis (Fig. 10j). There were only 6

575 DEGs in microglia from retinas treated with NMDA \pm BMS, consistent with the co576 clustering of microglia from these treatments in UMAP plots (Fig. 10d). These microglia 577 were harvested at 48hrs after NMDA-treatment and, thus, it is likely that significant 578 differences in gene expression that led to decreased accumulation of reactive microglia 579 in BMS/NMDA-treated retinas occurred shortly after damage and BMS-treatment. This 580 is consistent with scRNA-seq findings in NMDA-damaged mouse retinas wherein 581 microglia significantly up-regulated genes for pro-inflammatory cytokines between 3 and 58212 hrs after damage (Todd et al., 2019).

FASN influences MGPCs, neuronal survival and the accumulation of reactive microglia

Fatty acid synthase (FASN) - dependent fatty acid synthesis is necessary for

587 FABP activity. scRNA-seq data indicated that FASN is widely expressed by most retinal 588 cell types (Fig. 11a). In MG, levels of FASN were elevated in resting MG and down589 regulated in MG at 24, 48 and 72 hrs after NMDA treatment, and remained low and 590 prevalent in MGPCs (Fig. 11a,b). Relative levels of FASN in MG and MGPCs across 591 different treatments were highest in resting MG, lowest in MGPCs and intermediate 592 levels in activated MG at different times after NMDA and doses of insulin + FGF2 593 (supplemental Fig. 2f-h). We further assessed patterns of expression of FASN in 594 aggregate libraries from time-points soon after NMDA, at 3 and $12 \mathrm{hrs}$ after treatment. 595 Levels of FASN were significantly up-regulated in MG at 3hrs after NMDA and back 
596 down at 12 and $48 \mathrm{hrs}$ after NMDA (Fig. 11c,d), suggesting a rapid and transient need

597 for elevated fatty acid synthesis in MG shortly after NMDA-treatment.

Treatment of NMDA-damaged retinas with FASN inhibitors, G28UCM and C75, resulted in significant reductions in numbers of proliferating MGPCs (Fig. 11e-g). These large decreases in numbers of proliferating MGPCs occurred despite significant decreases in cell death (Fig. 11h-j) and increases in proliferation of microglia (Fig. 11k-

602 p) in NMDA-damaged retinas treated with FASN inhibitors. Increases in numbers of 603 proliferating microglia occurred without increasing total numbers of CD45+ cells (Fig.

$60411 \mathrm{k}, \mathrm{p})$, suggesting that there was less recruitment of peripheral monocytes coincident 605 with increased proliferation of resident microglia to result in no net change in total 606 numbers of CD45+ cells in the retina. NIRG cells were not significantly affected by 607 FASN inhibitors (not shown).

608 We next sought to investigate whether cell signaling in damaged retinas was 609 influenced by FASN-inhibitor. We observed a significant decrease in levels of pS6 in the cytoplasm of MG treated with FASN-inhibitor in damaged retinas (Fig. 12a,b). By comparison, levels of pStat3 and pSmad1/5/8 were undetectable in the nuclei of MG

612 treated with FASN-inhibitor in damaged retinas $(\mathrm{n} \geq 5)$ (Fig.12a). Signaling though mTor 613 (pS6), Jak/Stat- and BMP/SMAD-signaling are known to be required for the formation of 614 proliferating MGPCs (Todd et al., 2016a; Todd et al., 2017; Zelinka et al., 2016).

615 Inhibition of FASN in NMDA-damaged retinas had no effect upon numbers of TUNEL616 positive dying cells (Fig. 12a,d).

Discussion:

In this study we investigate the function of FASN and FABPs in glial cells in the chick retina. We observed that $F A B P 7$ is highly expressed by eRPCs and maturing MG during embryonic development. FABP7, FABP5 and PMP2 are up-regulated during the 622 activation of MG after damage or treatment with FGF2 and insulin. This pattern of FABP 623 expression was also observed for NIRG cells and microglia in damaged retinas.

624 Inhibition of FABPs or FASN influenced the proliferation of different types of cells 625 including NIRG cells, microglia, and MGPCs. Inhibition of FABPs in undamaged retinas induced reactivity in MG, but also both decreased levels of genes associated with 
resting mature MG and neurogenesis and increased genes associated with gliogenesis and inflammation. Inhibition of FABPs and FASN in damaged retinas selectively suppressed cell signaling pathways in MG that are known to promote the formation of MGPCs. These findings indicate the importance of FASN and FABPs in mediating the transition into a proliferating MGPCs.

FABPs in retinal development

Different FABP isoforms are known to be expressed by different cell types in maturing mammalian brain (Owada, 2008). Similarly, FABP isoforms have been identified in the chick retina (Sellner, 1993). FABP7 is often used as a biomarker for radial glia (brain lipid binding protein, Blbp) in the developing mouse brain and is presumed to facilitate cortical development (Anthony et al., 2005; Feng et al., 1994). FABPs are expressed in different types of tumors, particularly in cancer metastasis (Ohmachi et al., 2006; Senga et al., 2018). We found that FABP7 was upregulated in developing and maturing MG in embryonic chick retina. In addition, FABP7 was detected in developing amacrine and bipolar interneurons, but was downregulated in mature neurons. By comparison, FABP5 was expressed at high levels in mature interneurons. These cell-type specific patterns of expression for FABPs may indicate isoform-specific rolls despite overlap in ligand-binding among FABPs.

The chick eye is known to have proliferating retinal progenitor cells at the ciliary marginal zone of the retina (Fischer and Reh, 2000; Fischer et al., 2014a). Inhibition of FABPs did not influence the proliferation of $\mathrm{CMZ}$ progenitors. These progenitors expressed relatively low levels of PMP2, but expression of FABP5 and FABP7 in these cells remains unknown. Treatment with insulin and FGF2 upregulated PMP2 in MG and

652 progenitors. It is possible that FABP inhibitor had no effect because the drug failed to 653 diffuse through the vitreous to act at the CMZ. Alternatively, FABPs do influence the 654 proliferation of $\mathrm{CMZ}$ progenitors. Although many cell signaling pathways can influence 655 both CMZ progenitors and MGPCs, there are instances where CMZ progenitors and 656 MGPCs are differentially influenced by cell signaling pathways. For example, insulin and 
658 and Reh, 2002), whereas the insulin and IGF1 must be combined with FGF2 to 659 stimulate the proliferation of MGPCs (Fischer et al., 2002; Ritchey et al., 2012).

660 Similarly, HG-EGF stimulates the proliferation of MGPCs, but has no effect upon CMZ

661 progenitors even when combined with IGF1 (Todd et al., 2015). By comparison, 662 glucagon suppresses the proliferation of CMZ progenitors (Fischer et al., 2005), 663 whereas glucagon has no effect upon the proliferation of MGPCs (unpublished 664 observations). By contrast, the proliferation of CMZ progenitors and MGPCs can be 665 stimulated by Sonic Hedgehog signaling (Moshiri et al., 2005; Todd and Fischer, 2015), stimulated by retinoic acid (Todd et al., 2018) and by inhibition of Smad3 (Todd et al.,

667 2017).

\section{NIRG and Oligodendrocyte proliferate after damage}

During embryonic development glial precursor cells migrate into the retina from the optic nerve (Rompani and Cepko, 2010). These precursor cells undergo a cell division to generate oligodendrocytes and diacytes (Rompani and Cepko, 2010), also known as non-astrocytic inner retinal glia (NIRG) cell that reside predominantly in the IPL (Fischer et al., 2010). This unique type of glial cell has been identified in the retinas of birds and some types of reptiles (Todd et al., 2016b). The functions of the NIRG cells is unknown, but these cells are known to proliferate in response to IGF1 (Fischer et al., 2010) and their survival is tethered to retinal microglia (Zelinka et al., 2012b). We find that NIRG cells express PMP2, but only in peripheral regions of the retina or at later times ( $>7$ days) after NMDA-induced damage. By comparison, inhibition of FASN had no effect upon the proliferation of NIRG cells.

Consistent with previous reports (Kohsaka et al., 1980; Kohsaka et al., 1983), the chicken retina contains axons that are thinly myelinated by oligodendrocytes that

683 express Sox10, Olig2 and PMP2. Surprisingly, we observed that these oligodendrocytes 684 express HuC/D, a common neuronal marker of amacrine cells and ganglion cells. Thus, unambiguous identification of neurons in the GCL requires markers in addition to

$686 \mathrm{HuC} / \mathrm{D}$. There were rare PMP2 ${ }^{+}$cell in the inner INL of peripheral regions of retina that 687 did not express neuronal markers, but expressed a set of markers associated with NIRG cells. One week after damage there was an increase in the number of EdU- 
labeled oligodendrocytes, which suggests de novo myelination of axons in the NFL from newly generated oligodendrocytes. Further studies are required to determine whether the newly generated oligodendrocytes directly results in additional axon myelination. Notably, NMDA-damage is not expected to result in demyelination, which raises questions about the signals that promote the proliferation of oligodendrocytes. Without genetic models or viruses with appropriate trophism to lineage-trace the origin of newly generated oligodendrocytes, the origin of oligodendrocyte precursor cells remains uncertain.

\section{The formation of proliferating MGPCs requires FABPs and FASN}

scRNA-seq data indicate that $F A B P 7$ is expressed by resting MG in the postnatal chick retina. When the retina is damaged or treated with FGF2 and insulin the MG robustly upregulate FABP5, FABP7 and PMP2. When FABPs are inhibited in damaged retinas, significantly fewer proliferating MGPCs are generated (Hoang et al., 2020). Similarly, MGPC proliferation was suppressed when inhibitor was applied before damage when only FABP7 is highly expressed in MG. We observed diminished cell signaling through pSMAD1/5/8, pStat3, and mTor (pS6) in MG treated with FABP inhibitor. These findings are consistent with treatment-specific Ligand-Receptor interactions in glia treated by FABP inhibitor revealed by SingleCellSignalR analyses. Similarly, levels of pSMAD1/5/8 and pStat3 fell below detection in MG treated with FASN-inhibitor. These cell signaling pathways have been shown to promote the formation of proliferating MGPCs in the chick retina (Todd et al., 2016a; Todd et al., 2017). Further, we find loss of Ligand-Receptor interactions involving FGF, Midkine and

712 Notch1 in retinal glia treated with FABP inhibitor. (Anthony et al., 2005). These

713 pathways are necessary the formation of proliferating MGPCs (Fischer et al., 2009a;

714 Fischer et al., 2009b; Ghai et al., 2010; Hayes et al., 2007). Consistent with our

715 observations, Notch-signaling is known to be downstream of FABPs (Anthony et al., 716 2005).

FABP isoforms serve many different functions including cellular metabolism and 718 cellular trafficking of lipid metabolites (Storch and Corsico, 2008). In addition to 
720 ligands for cell signaling such as PPAR (Tripathi et al., 2017), retinoic acid (Dawson and Xia, 2012), and endocannabinoids (Haj-Dahmane et al., 2018). Given the wellestablished involvement of FABPs in lipid metabolism, we found associations with the expression of FASN, which is important for producing long chain fatty acids (Kuhajda, 2006). When we antagonize FASN with different small molecule inhibitors there are significant decreases in numbers of proliferating MGPCs. These findings provide further evidence that lipid metabolism is required for the transition of resting MG to activated states, and subsequent proliferation as progenitor-like cells. scRNA-seq data indicate that MG become reactive with FABP inhibition in the absence of neuronal damage; there was significant upregulation of genes associated with gliogenesis and reactivity. This broad shift in the gene-expression modules to induce reactive phenotypes was supported by evidence for Ligand-Receptor interactions associated with reactivity including signals such as IL1 $\beta$, TGF $\beta$ and HB-EGF.

Although inhibition of FABPs in undamaged retinas stimulated MG to adopt a reactive phenotype and acquire a transcriptomic profile characteristic of activation and de-differentiation, FABP-inhibition prior to NMDA did not prime MG to become MGPCs. This likely resulted from FABP-inhibition up-regulating gene modulates associated with gliogenesis and down-regulation of gene modulates associated with neurogenesis and proliferation. FABP-inhibition in undamaged retinas should have inhibited FABP7 in resting MG and PMP2 in oligodendocytes, and this inhibition may have resulted in the activated transcriptomic profiles seen in MG and microglia. However, treatment with FABP inhibitor resulted in an upregulation of FABP5 and PMP2 in MG and FABP5, FABP7 and PMP2 in microglia. Thus, it is possible that the suppressed formation of

743 MGPCs following FABP inhibition resulted from upregulation and inhibition of FABPs in

744 MG and microglia.

\section{FABP-inhibition suppresses proliferation and induces reactivity in microglia}

The development of FABP inhibitors was motivated by the presence of FABP4 in

748 obese patients suffering from atherosclerosis, where macrophages contribute the 749 narrowing of arterial vessels (Furuhashi et al., 2007; Makowski et al., 2001). Peripheral macrophages express FABP4 and inhibition may slow the progression of vessel 
narrowing (Furuhashi et al., 2007; Makowski et al., 2001). After retinal damage in chick,

752 microglia normally proliferate and acquire a reactive phenotype (Zelinka et al., 2012b).

753 The presence of reactive microglia is required for the formation of MGPCs (Fischer et

754 al., 2014b). Further, signals from reactive microglia mediate inflammatory signaling in

755 MG through pathways such as NFkB (Palazzo et al., 2020). Given that microglia rapidly

756 respond to retinal damage with upregulation of pro-inflammatory signals (Todd et al.,

757 2019), it is possible that reduced numbers of microglia in damaged retinas treated with

758 FABP inhibitor also influenced the formation of proliferating MGPCs. In damaged retinas

759 treated with FABP inhibitor, microglia appeared highly reactive, but the numbers of

760 these cells were significantly reduced. Further, we did not detect significant numbers of

761 microglial genes that were differentially expressed in damaged retinas treated with

762 FABP inhibitor. Thus, it seems most likely that microglia did not influence the formation

763 of MGPCs in inhibitor-treated retinas.

Conclusions:

FASN and FABPs are novel targets of investigation with respect to retinal glia and reprogramming of MG into MGPCs. We found that FABPs are highly expressed by MG during reprogramming into proliferating MGPCs. Inhibition of FABPs results in the upregulation of genes associated with gliogenesis and inflammation while concurrently reducing the expression of genes associated with proliferation and neurogenesis. The anti-proliferative effects of FABP inhibition were not specific to MG, as microglia also showed reduced proliferation in inhibitor-treated retinas. By contrast, the proliferation of $\mathrm{CMZ}$ was unaffected by FABP inhibitor. Our findings suggest that FABPs mediate glial

774 reactivity and de-differentiation through lipid-associated cell signaling, while proliferation 775 requires lipid metabolism. Consistent with this hypothesis, inhibition of FASN potently

776 inhibited the formation of proliferating MGPCs, while decreasing cell death and

777 increasing microglial proliferation. Microglia express FABPs, and FABP inhibition alters

778 cytokine production and reactivity which is expected to impact signaling with MG.

779 Collectively, our data suggest the activity of FASN and FABPs MG to become activated 780 prior to forming proliferating MGPCs in the chick retina. 
783 Author contributions: WAC - experimental design, execution of experiments,

784 collection of data, data analysis, construction of figures and writing the manuscript. AT,

$785 \mathrm{EH}$ and $\mathrm{MH}$ - execution of experiments and collection of data. $\mathrm{HE}$ - experimental

786 design, collection of data, data analysis and writing the manuscript. TH and SB -

787 preparation of scRNA-seq libraries. AJF - experimental design, data analysis,

788 construction of figures and writing the manuscript.

789

790 Data availability: RNA-Seq data for gene-cell matrices are deposited at GitHub

791 https://github.com/jiewwwang/Single-cell-retinal-regeneration

792 https://github.com/fischerlab3140/scRNAseq libraries

793 Some of the scRNA-seq data can be queried at

794 https://proteinpaint.stjude.org/F/2019.retina.scRNA.html. 
References:

Allen, J. A., Halverson-Tamboli, R. A. and Rasenick, M. M. (2007). Lipid raft microdomains and neurotransmitter signalling. Nat. Rev. Neurosci. 8, 128-140.

Anthony, T. E., Mason, H. A., Gridley, T., Fishell, G. and Heintz, N. (2005). Brain lipidbinding protein is a direct target of Notch signaling in radial glial cells. Genes Dev. 19, 1028-1033.

Bernardos, R. L., Barthel, L. K., Meyers, J. R. and Raymond, P. A. (2007). Late-stage neuronal progenitors in the retina are radial Muller glia that function as retinal stem cells. J Neurosci 27, 7028-40.

Blackshaw, S. and Sanes, J. R. (2021). Turning lead into gold: reprogramming retinal cells to cure blindness. J. Clin. Invest. 131,.

Bogdan, D., Falcone, J., Kanjiya, M. P., Park, S. H., Carbonetti, G., Studholme, K., Gomez, M., Lu, Y., Elmes, M. W., Smietalo, N., et al. (2018). Fatty acid-binding protein 5 controls microsomal prostaglandin E synthase 1 (mPGES-1) induction during inflammation. J. Biol. Chem. 293, 5295-5306.

Butler, A., Hoffman, P., Smibert, P., Papalexi, E. and Satija, R. (2018). Integrating single-cell transcriptomic data across different conditions, technologies, and species. Nat. Biotechnol. 36, 411-420.

Cabello-Aguilar, S., Alame, M., Kon-Sun-Tack, F., Fau, C., Lacroix, M. and Colinge, J. (2020). SingleCellSignalR: inference of intercellular networks from single-cell transcriptomics. Nucleic Acids Res. 48, e55.

Campbell, W. A., Fritsch-Kelleher, A., Palazzo, I., Hoang, T., Blackshaw, S. and Fischer, A. J. (2021a). Midkine is neuroprotective and influences glial reactivity and the formation of Müller glia-derived progenitor cells in chick and mouse retinas. Glia 69, 1515-1539.

Campbell, W. A., Blum, S., Reske, A., Hoang, T., Blackshaw, S. and Fischer, A. J. (2021b). Cannabinoid signaling promotes the de-differentiation and proliferation of Müller gliaderived progenitor cells. Glia.

Dawson, M. I. and Xia, Z. (2012). The retinoid X receptors and their ligands. Biochim. Biophys. Acta 1821, 21-56.

Fausett, B. V. and Goldman, D. (2006). A role for alpha1 tubulin-expressing Muller glia in regeneration of the injured zebrafish retina. J Neurosci 26, 6303-13.

Fausett, B. V., Gumerson, J. D. and Goldman, D. (2008). The proneural basic helix-loop-helix gene ascl1a is required for retina regeneration. J. Neurosci. Off. J. Soc. Neurosci. 28, 1109-1117.

Feng, L., Hatten, M. E. and Heintz, N. (1994). Brain lipid-binding protein (BLBP): A novel signaling system in the developing mammalian CNS. Neuron 12, 895-908.

Fischer, A. J. and Bongini, R. (2010). Turning Müller glia into neural progenitors in the retina. Mol. Neurobiol. 42, 199-209. 
Fischer, A. J. and Reh, T. A. (2000). Identification of a proliferating marginal zone of retinal progenitors in postnatal chickens. Dev. Biol. 220, 197-210.

Fischer, A. J. and Reh, T. A. (2001). Müller glia are a potential source of neural regeneration in the postnatal chicken retina. Nat. Neurosci. 4, 247-252.

Fischer, A. J. and Reh, T. A. (2002). Exogenous growth factors stimulate the regeneration of ganglion cells in the chicken retina. Dev. Biol. 251, 367-379.

Fischer, A. J. and Reh, T. A. (2003). Potential of Muller glia to become neurogenic retinal progenitor cells. Glia 43, 70-6.

Fischer, A. J., Seltner, R. L. P., Poon, J. and Stell, W. K. (1998). Immunocytochemical characterization of quisqualic acid-and $\mathrm{N}$-methyl-D-aspartate-induced excitotoxicity in the retina of chicks. J. Comp. Neurol. 393, 1-15.

Fischer, A. J., McGuire, C. R., Dierks, B. D. and Reh, T. A. (2002). Insulin and fibroblast growth factor 2 activate a neurogenic program in Müller glia of the chicken retina. $J$. Neurosci. Off. J. Soc. Neurosci. 22, 9387-9398.

Fischer, A. J., Omar, G., Walton, N. A., Verrill, T. A. and Unson, C. G. (2005). Glucagonexpressing neurons within the retina regulate the proliferation of neural progenitors in the circumferential marginal zone of the avian eye. J. Neurosci. Off. J. Soc. Neurosci. 25, 10157-10166.

Fischer, A. J., Skorupa, D., Schonberg, D. L. and Walton, N. A. (2006). Characterization of glucagon-expressing neurons in the chicken retina. J. Comp. Neurol. 496, 479-494.

Fischer, A. J., Foster, S., Scott, M. A. and Sherwood, P. (2008). The transient expression of LIM-domain transcription factors is coincident with the delayed maturation of photoreceptors in the chicken retina. J. Comp. Neurol. 506, 584-603.

Fischer, A. J., Scott, M. A. and Tuten, W. (2009a). Mitogen-activated protein kinase-signaling stimulates Müller glia to proliferate in acutely damaged chicken retina. Glia 57, 166-181.

Fischer, A. J., Scott, M. A., Ritchey, E. R. and Sherwood, P. (2009b). Mitogen-activated protein kinase-signaling regulates the ability of Müller glia to proliferate and protect retinal neurons against excitotoxicity. Glia 57, 1538-1552.

Fischer, A. J., Scott, M. A., Zelinka, C. and Sherwood, P. (2010). A novel type of glial cell in the retina is stimulated by insulin-like growth factor 1 and may exacerbate damage to neurons and Muller glia. Glia 58, 633-49.

Fischer, A. J., Bosse, J. L. and El-Hodiri, H. M. (2014a). Reprint of: the ciliary marginal zone (CMZ) in development and regeneration of the vertebrate eye. Exp. Eye Res. 123, 115120.

Fischer, A. J., Zelinka, C., Gallina, D., Scott, M. A. and Todd, L. (2014b). Reactive microglia and macrophage facilitate the formation of Müller glia-derived retinal progenitors. Glia 62, 1608-1628. 
873

874

875

876

877

878

879

880

881

882

883

884

885

886

887

888

889

890

891

892

893

894

895

896

897

898

899

900

901

902

903

904

905

906

907

908

909

Furuhashi, M., Tuncman, G., Görgün, C. Z., Makowski, L., Atsumi, G., Vaillancourt, E., Kono, K., Babaev, V. R., Fazio, S., Linton, M. F., et al. (2007). Treatment of diabetes and atherosclerosis by inhibiting fatty-acid-binding protein aP2. Nature 447, 959-965.

Gallina, D., Todd, L. and Fischer, A. J. (2014a). A comparative analysis of Müller gliamediated regeneration in the vertebrate retina. Exp. Eye Res. 123, 121-130.

Gallina, D., Zelinka, C. and Fischer, A. J. (2014b). Glucocorticoid receptors in the retina, Müller glia and the formation of Müller glia-derived progenitors. Dev. Camb. Engl. 141, 3340-3351.

Ghai, K., Zelinka, C. and Fischer, A. J. (2009). Serotonin released from amacrine neurons is scavenged and degraded in bipolar neurons in the retina. J. Neurochem. 111, 1-14.

Ghai, K., Zelinka, C. and Fischer, A. J. (2010). Notch signaling influences neuroprotective and proliferative properties of mature Müller glia. J. Neurosci. Off. J. Soc. Neurosci. 30, 3101-3112.

Haj-Dahmane, S., Shen, R.-Y., Elmes, M. W., Studholme, K., Kanjiya, M. P., Bogdan, D., Thanos, P. K., Miyauchi, J. T., Tsirka, S. E., Deutsch, D. G., et al. (2018). Fatty-acidbinding protein 5 controls retrograde endocannabinoid signaling at central glutamate synapses. Proc. Natl. Acad. Sci. 115, 3482-3487.

Hamburger, V. and Hamilton, H. L. (1992). A series of normal stages in the development of the chick embryo. 1951. Dev Dyn 195, 231-72.

Hamon, A., García-García, D., Ail, D., Bitard, J., Chesneau, A., Dalkara, D., Locker, M., Roger, J. E. and Perron, M. (2019). Linking YAP to Müller Glia Quiescence Exit in the Degenerative Retina. Cell Rep. 27, 1712-1725.e6.

Hanhoff, T., Lücke, C. and Spener, F. (2002). Insights into binding of fatty acids by fatty acid binding proteins. Mol. Cell. Biochem. 239, 45-54.

Hayes, S., Nelson, B. R., Buckingham, B. and Reh, T. A. (2007). Notch signaling regulates regeneration in the avian retina. Dev Bio/312, 300-11.

Hitchcock, P. F. and Raymond, P. A. (1992). Retinal regeneration. Trends Neurosci 15, 1038.

Hoang, T., Wang, J., Boyd, P., Wang, F., Santiago, C., Jiang, L., Yoo, S., Lahne, M., Todd, L. J., Jia, M., et al. (2020). Gene regulatory networks controlling vertebrate retinal regeneration. Science 370,.

Jorstad, N. L., Wilken, M. S., Grimes, W. N., Wohl, S. G., VandenBosch, L. S., Yoshimatsu, T., Wong, R. O., Rieke, F. and Reh, T. A. (2017). Stimulation of functional neuronal regeneration from Muller glia in adult mice. Nature.

Karl, M. O., Hayes, S., Nelson, B. R., Tan, K., Buckingham, B. and Reh, T. A. (2008). Stimulation of neural regeneration in the mouse retina. Proc Natl Acad Sci U A 105, 19508-13. 
Kohsaka, S., Takamatsu, K., Nishimura, Y., Mikoshiba, K. and Tsukada, Y. (1980). Neurochemical characteristics of myelin-like structure in the chick retina. $J$ Neurochem 34, 662-8.

Kohsaka, S., Nishimura, Y., Takamatsu, K., Shimai, K. and Tsukada, Y. (1983). Immunohistochemical localization of 2',3'-cyclic nucleotide 3'-phosphodiesterase and myelin basic protein in the chick retina. J. Neurochem. 41, 434-439.

Kuhajda, F. P. (2006). Fatty acid synthase and cancer: new application of an old pathway. Cancer Res. 66, 5977-5980.

Li, B., Zhang, G., Li, C., He, D., Li, X., Zhang, C., Tang, F., Deng, X., Lu, J., Tang, Y., et al. (2012). Identification of JAK2 as a mediator of FIP1L1-PDGFRA-induced eosinophil growth and function in CEL. PloS One 7, e34912.

Makowski, L., Boord, J. B., Maeda, K., Babaev, V. R., Uysal, K. T., Morgan, M. A., Parker, R. A., Suttles, J., Fazio, S., Hotamisligil, G. S., et al. (2001). Lack of macrophage fatty-acid-binding protein aP2 protects mice deficient in apolipoprotein $\mathrm{E}$ against atherosclerosis. Nat. Med. 7, 699-705.

Moshiri, A., McGuire, C. R. and Reh, T. A. (2005). Sonic hedgehog regulates proliferation of the retinal ciliary marginal zone in posthatch chicks. Dev Dyn 233, 66-75.

Ohmachi, T., Inoue, H., Mimori, K., Tanaka, F., Sasaki, A., Kanda, T., Fujii, H., Yanaga, K. and Mori, M. (2006). Fatty Acid Binding Protein 6 Is Overexpressed in Colorectal Cancer. Clin. Cancer Res. 12, 5090-5095.

Ooto, S., Akagi, T., Kageyama, R., Akita, J., Mandai, M., Honda, Y. and Takahashi, M. (2004). Potential for neural regeneration after neurotoxic injury in the adult mammalian retina. Proc Natl Acad Sci U A 101, 13654-9.

Owada, Y. (2008). Fatty acid binding protein: localization and functional significance in the brain. Tohoku J. Exp. Med. 214, 213-220.

Palazzo, I., Deistler, K., Hoang, T. V., Blackshaw, S. and Fischer, A. J. (2020). NF-KB signaling regulates the formation of proliferating Müller glia-derived progenitor cells in the avian retina. Development 147 ,.

Peng, X., Studholme, K., Kanjiya, M. P., Luk, J., Bogdan, D., Elmes, M. W., Carbonetti, G., Tong, S., Gary Teng, Y.-H., Rizzo, R. C., et al. (2017). Fatty-acid-binding protein inhibition produces analgesic effects through peripheral and central mechanisms. Mol. Pain 13, 1744806917697007.

Pollak, J., Wilken, M. S., Ueki, Y., Cox, K. E., Sullivan, J. M., Taylor, R. J., Levine, E. M. and Reh, T. A. (2013). Ascl1 reprograms mouse Muller glia into neurogenic retinal progenitors. Development 140, 2619-2631.

Popielarczyk, T. L., Huckle, W. R. and Barrett, J. G. (2019). Human Bone Marrow-Derived Mesenchymal Stem Cells Home via the PI3K-Akt, MAPK, and Jak/Stat Signaling Pathways in Response to Platelet-Derived Growth Factor. Stem Cells Dev. 28, 11911202. 
Qiu, X., Hill, A., Packer, J., Lin, D., Ma, Y.-A. and Trapnell, C. (2017a). Single-cell mRNA quantification and differential analysis with Census. Nat. Methods 14, 309-315.

Qiu, X., Mao, Q., Tang, Y., Wang, L., Chawla, R., Pliner, H. A. and Trapnell, C. (2017b). Reversed graph embedding resolves complex single-cell trajectories. Nat. Methods 14, 979-982.

Raymond, P. A. (1991). Retinal regeneration in teleost fish. Ciba Found Symp 160, 171-86; discussion 186-91.

Reichenbach, A. and Bringmann, A. (2013). New functions of Müller cells. Glia 61, 651-678.

Ritchey, E. R., Zelinka, C. P., Tang, J., Liu, J. and Fischer, A. J. (2012). The combination of IGF1 and FGF2 and the induction of excessive ocular growth and extreme myopia. Exp. Eye Res. 99, 1-16.

Rompani, S. B. and Cepko, C. L. (2010). A Common Progenitor for Retinal Astrocytes and Oligodendrocytes. J. Neurosci. 30, 4970-4980.

Rueda, E. M., Hall, B. M., Hill, M. C., Swinton, P. G., Tong, X., Martin, J. F. and Poché, R. A. (2019). The Hippo Pathway Blocks Mammalian Retinal Müller Glial Cell Reprogramming. Cell Rep. 27, 1637-1649.e6.

Satija, R., Farrell, J. A., Gennert, D., Schier, A. F. and Regev, A. (2015). Spatial reconstruction of single-cell gene expression data. Nat Biotechno/ 33, 495-502.

Sellner, P. A. (1993). Retinal FABP principally localizes to neurons and not to glial cells. Mol. Cell. Biochem. 123, 121-127.

Sellner, P. A., Chu, W., Glatz, J. F. and Berman, N. E. (1995). Developmental role of fatty acid-binding proteins in mouse brain. Brain Res. Dev. Brain Res. 89, 33-46.

Senga, S., Kawaguchi, K., Kobayashi, N., Ando, A. and Fujii, H. (2018). A novel fatty acidbinding protein 5-estrogen-related receptor a signaling pathway promotes cell growth and energy metabolism in prostate cancer cells. Oncotarget 9, 31753-31770.

Smathers, R. L. and Petersen, D. R. (2011). The human fatty acid-binding protein family: evolutionary divergences and functions. Hum. Genomics 5, 170-191.

Storch, J. and Corsico, B. (2008). The Emerging Functions and Mechanisms of Mammalian Fatty Acid-Binding Proteins. Annu. Rev. Nutr. 28, 73-95.

Todd, L. and Fischer, A. J. (2015). Hedgehog-signaling stimulates the formation of proliferating Müller glia-derived progenitor cells in the retina. Development 142, 26102622.

Todd, L., Volkov, L. I., Zelinka, C., Squires, N. and Fischer, A. J. (2015). Heparin-binding EGF-like growth factor (HB-EGF) stimulates the proliferation of Müller glia-derived progenitor cells in avian and murine retinas. Mol. Cell. Neurosci. 69, 54-64. 
984

985

986

987

988

989

990

991

992

993

994

995

996

997

998

999

1000

1001

1002

1003

1004

1005

1006

1007

1008

1009

1010

1011

1012

1013

1014

1015

1016

1017

1018

1019

1020

1021

1022

Todd, L., Squires, N., Suarez, L. and Fischer, A. J. (2016a). Jak/Stat signaling regulates the proliferation and neurogenic potential of Müller glia-derived progenitor cells in the avian retina. Sci. Rep. 6, 35703.

Todd, L., Suarez, L., Squires, N., Zelinka, C. P., Gribbins, K. and Fischer, A. J. (2016b). Comparative analysis of glucagonergic cells, glia, and the circumferential marginal zone in the reptilian retina. J. Comp. Neurol. 524, 74-89.

Todd, L., Palazzo, I., Squires, N., Mendonca, N. and Fischer, A. J. (2017). BMP- and TGF $\beta$ signaling regulate the formation of Müller glia-derived progenitor cells in the avian retina. Glia 65, 1640-1655.

Todd, L., Suarez, L., Quinn, C. and Fischer, A. J. (2018). Retinoic Acid-Signaling Regulates the Proliferative and Neurogenic Capacity of Müller Glia-Derived Progenitor Cells in the Avian Retina. Stem Cells Dayt. Ohio 36, 392-405.

Todd, L., Palazzo, I., Suarez, L., Liu, X., Volkov, L., Hoang, T. V., Campbell, W. A., Blackshaw, S., Quan, N. and Fischer, A. J. (2019). Reactive microglia and IL1ß/IL1R1-signaling mediate neuroprotection in excitotoxin-damaged mouse retina. $J$. Neuroinflammation 16, 118.

Todd, L., Finkbeiner, C., Wong, C. K., Hooper, M. J. and Reh, T. A. (2020). Microglia Suppress Ascl1-Induced Retinal Regeneration in Mice. Cell Rep. 33, 108507.

Trapnell, C., Roberts, A., Goff, L., Pertea, G., Kim, D., Kelley, D. R., Pimentel, H., Salzberg, S. L., Rinn, J. L. and Pachter, L. (2012). Differential gene and transcript expression analysis of RNA-seq experiments with TopHat and Cufflinks. Nat Protoc 7, 562-78.

Tripathi, S., Kushwaha, R., Mishra, J., Gupta, M. K., Kumar, H., Sanyal, S., Singh, D., Sanyal, S., Sahasrabuddhe, A. A., Kamthan, M., et al. (2017). Docosahexaenoic acid up-regulates both PI3K/AKT-dependent FABP7-PPARY interaction and MKP3 that enhance GFAP in developing rat brain astrocytes. J. Neurochem. 140, 96-113.

Ueki, Y., Wilken, M. S., Cox, K. E., Chipman, L., Jorstad, N., Sternhagen, K., Simic, M., Ullom, K., Nakafuku, M. and Reh, T. A. (2015). Transgenic expression of the proneural transcription factor Ascl1 in Muller glia stimulates retinal regeneration in young mice. Proc Natl Acad Sci U A 112, 13717-22.

Wan, J. and Goldman, D. (2016). Retina regeneration in zebrafish. Curr Opin Genet Dev 40, 41-47.

Wan, J., Ramachandran, R. and Goldman, D. (2012). HB-EGF is necessary and sufficient for Muller glia dedifferentiation and retina regeneration. Dev Cell 22, 334-47.

White, D. T., Sengupta, S., Saxena, M. T., Xu, Q., Hanes, J., Ding, D., Ji, H. and Mumm, J. S. (2017). Immunomodulation-accelerated neuronal regeneration following selective rod photoreceptor cell ablation in the zebrafish retina. Proc. Natl. Acad. Sci. U. S. A. 114, E3719-E3728.

Yamashima, T. (2012). "PUFA-GPR40-CREB signaling" hypothesis for the adult primate neurogenesis. Prog. Lipid Res. 51, 221-231. 
Yao, K., Qiu, S., Wang, Y. V., Park, S. J. H., Mohns, E. J., Mehta, B., Liu, X., Chang, B., Zenisek, D., Crair, M. C., et al. (2018). Restoration of vision after de novo genesis of rod photoreceptors in mammalian retinas. Nature 560, 484-488.

Zelinka, C. P., Scott, M. A., Volkov, L. and Fischer, A. J. (2012a). The reactivity, distribution and abundance of Non-astrocytic Inner Retinal Glial (NIRG) cells are regulated by microglia, acute damage, and IGF1. PloS One 7, e44477.

1029

1030

1031

Zelinka, C. P., Scott, M. A., Volkov, L. and Fischer, A. J. (2012b). The Reactivity, Distribution and Abundance of Non-Astrocytic Inner Retinal Glial (NIRG) Cells Are Regulated by Microglia, Acute Damage, and IGF1. PLOS ONE 7, e44477.

Zelinka, C. P., Volkov, L., Goodman, Z. A., Todd, L., Palazzo, I., Bishop, W. A. and Fischer, A. J. (2016). mTor signaling is required for the formation of proliferating Müller gliaderived progenitor cells in the chick retina. Dev. Camb. Engl. 143, 1859-1873.

1035 
Table 1. List of antibodies, working dilution, clone/catalog number and source.

1038

\begin{tabular}{|l|l|l|l|l|}
\hline Antibody & Dilution & Host & $\begin{array}{l}\text { Clone/Catalog } \\
\text { number }\end{array}$ & Source \\
\hline Sox2 & $1: 1000$ & Goat & KOY0418121 & R\&D \\
\hline Sox9 & $1: 2000$ & Rabbit & AB5535 & Millipore \\
\hline Sox10 & $1: 500$ & Goat & PA5-47001 & Invitrogen \\
\hline CD45 & $1: 200$ & Mouse & HIS-C7 & Prionics \\
\hline Nkx2.2 & $1: 100$ & Mouse & 74.5A5 & DSHB \\
\hline PMP2 & $1: 100$ & Rabbit & 12717.AP & Proteintech \\
\hline AP2-alpha & $1: 50$ & Mouse & AP2A & DSHB \\
\hline Brn3a & $1: 50$ & Mouse & MAB1585 & Chemicon \\
\hline Islet1 & $1: 50$ & Mouse & 40.2D6 & DSHB \\
\hline Tyrosine hydroxylase & $1: 50$ & Mouse & aTH & DSHB \\
\hline Olig2 & $1: 50$ & Mouse & PCRP-Olig2-1E9 & DSHB \\
\hline Vimentin & $1: 50$ & Mouse & H5 & DSHB \\
\hline Glutamine synthetase & $1: 1000$ & Mouse & 610517 & BD Biosciences \\
\hline PS6 & $1: 750$ & Rabbit & \#2215; Ser240/244 & Cell Signaling \\
\hline PStat3 & $1: 100$ & Rabbit & 9131 & Cell Signaling \\
\hline pSmad1/5/8 & $1: 200$ & Rabbit & D5B10 & Cell Signaling \\
\hline & & & & \\
\hline
\end{tabular}

1039

1040

1041

1042

1043

1044

1045

1046

1047

1048

1049

1050

1051

1052

1053

1054

1055

1056

1057

Figure legends:

Figure 1. Expression of $F A B P 5, F A B P 7$ and $P M P 2$ in embryonic chick retina. scRNAseq libraries were generated from embryonic retinal cells at four stages of development (E5, E8, E12, E15) (a). UMAP clustered were generated to identify cell types and probe for gene expression (b). Cells were identified based on expression of cell-distinguishing genes $(\mathbf{a}, \mathbf{b})$. FABP isoforms were plotted in a heatmap, with cells expressing $2+$ genes denoted in black (c). The expression of FABPs in different maturing cell types is represented in violin plots (d). MG were also plotted in pseudotime denoting their transition from an immature progenitor cell to mature glial cell (e). FABP5 and FABP7 expression fluctuated during this transition as denoted by pseudotime scatter plots $(\mathbf{f})$ and pseudotime state violin plots $(\mathbf{g})$. Significant difference $\left({ }^{*} p<0.01,{ }^{* *} p<0.0001\right.$, ${ }^{* * *} \mathrm{p}<<0.0001$ ) was determined by using a Wilcox rank sum with Bonferoni correction. RPC - retinal progenitor cell, MG - Müller glia, iMG - immature Müller glia, mMG mature Müller glia. 

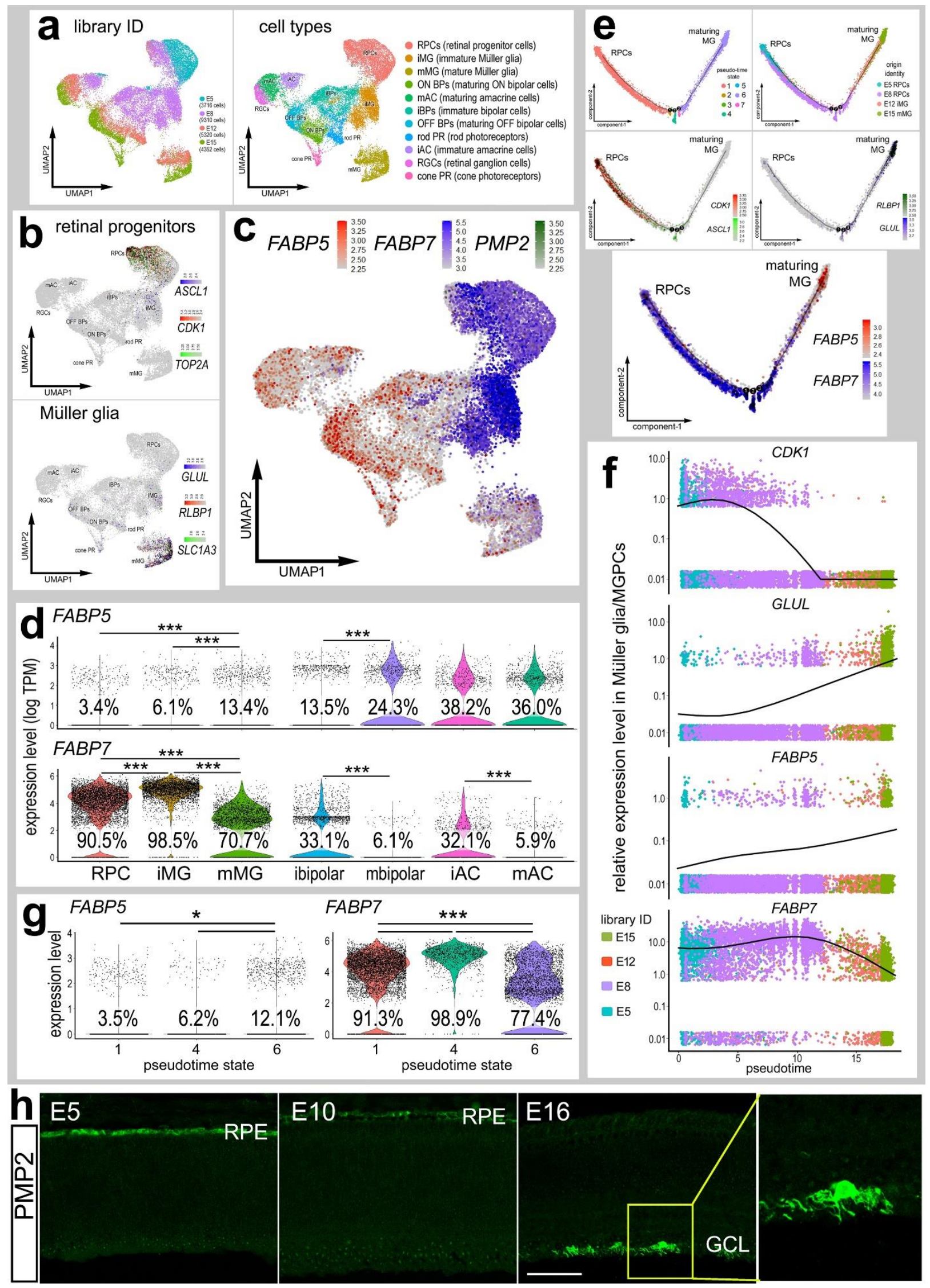
1060 Figure 2.Chick MG of damaged retinas express FABP isoforms preferentially during the 1061 MGPC transition. scRNA-seq libraries of time points after NMDA damage were 1062 aggregated and clustered with UMAP to identify unique MG clusters transitioning into 1063 MGPCs $(\mathbf{a}, \mathbf{b})$. The levels of FABP7, FABP5, PMP2 in MG clusters, oligodendrocytes, 1064 and nonastrocytic inner retinal glia (NIRG) cells are represented by violin plots (d). The 1065 response of MG to damage was modelled in pseudotime, indicating a divergent 1066 response between glial reactivity and de-differentiated MGPC (e). The expression levels 1067 of FABPs are compared between a "reactive" and "reprogramming" response by MG. 1068 FABP expression are shown in violin plots of MG in different transitional states (f). 1069 These divergent transitions are shown on the pseudotime scatter plot with the reactive 1070 and reprogramming branch are denoted by the black and red lines respectively $(\mathbf{g})$. 1071 Each dot in violin and scatter plots represents an individual cell. Significant changes $1072\left({ }^{*} p<0.1,{ }^{* *} p<0.0001,{ }^{* *} p<<0.0001\right)$ in levels were determined by using a Wilcox rank 1073 sum with Bonferroni correction. 


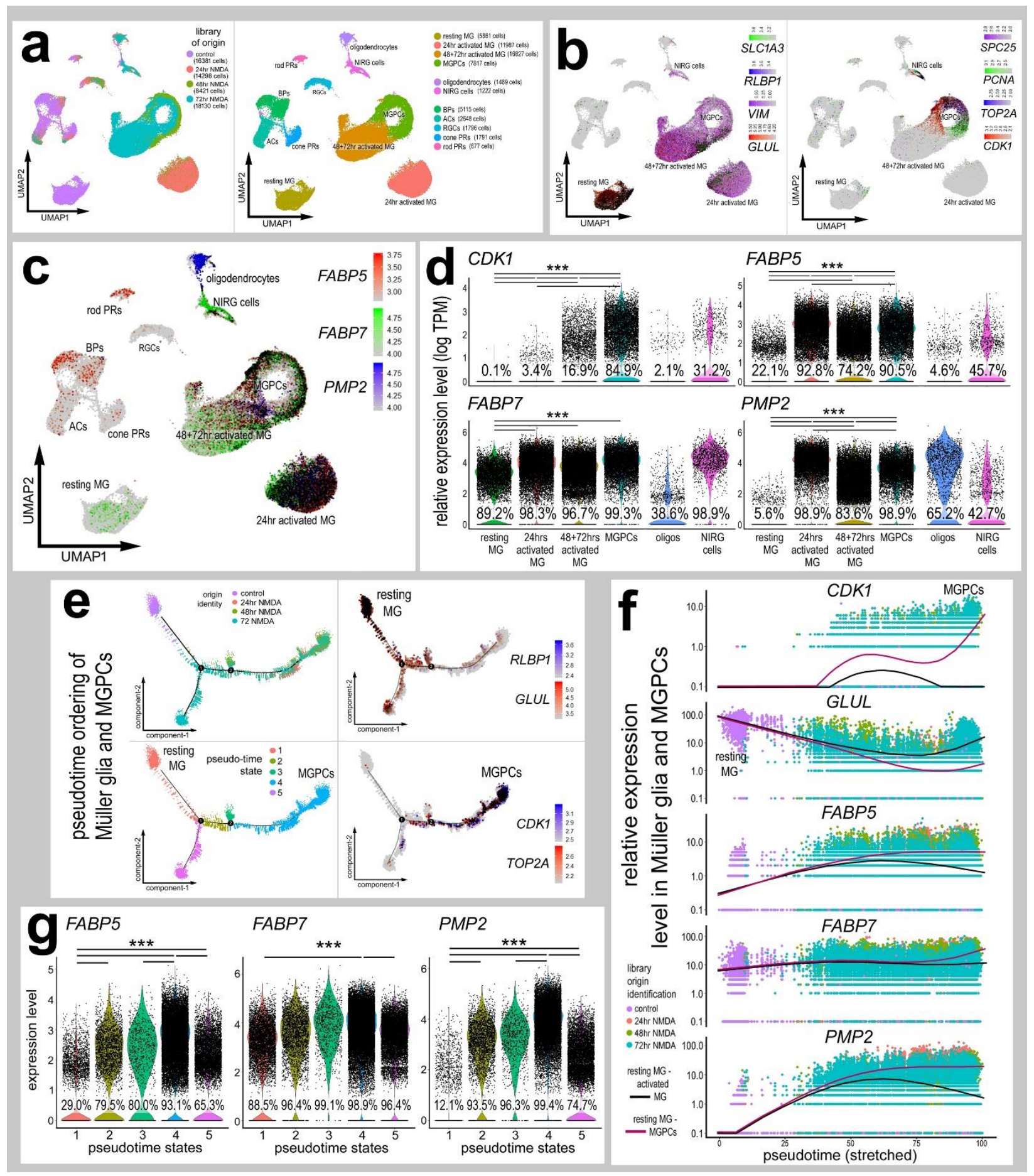


1079 Figure 3. PMP2-immunoreactivity in chick retinas. Vertical sections of the retina were 1080 obtained from untreated eyes (a-e, $\mathbf{g}, \mathbf{h})$ and eyes injected with NMDA (i). Tissues were 1081 labeled with antibodies to PMP2 (green) and Ap2a (red; a), Islet1 (red; a), tyrosine 1082 hydroxylase (red; a), GS (red; b), Olig2 (red, c,e), HuC/D (red; d), EdU (blue; g), Sox10 1083 (red; g), Sox2 (blue; h,i), and Nkx2.2 (red; h,i). Arrows indicate double-labeled cells. 1084 Histograms illustrate the mean ( \pm SD) number of EdU+/PMP2+ oligodendrocytes in 1085 central and peripheral regions of the retina. Each dot represents one biological 1086 replicate. Significance $\left({ }^{*} p<0.01\right)$ of difference was determined by using a Student's t1087 test. Abbreviations: ONL - outer nuclear layer, INL - inner nuclear layer, IPL - inner 1088 plexiform layer, GCL - ganglion cell layer, GS - glutamine synthetase. (j) scRNA-seq 1089 was used to verify patterns of immunolabeling in NIRG cells and oligodendrocytes.

1090 These cells were isolated with UMAP ordering maintained for libraries from control and 1091 NMDA-damaged retinas. Heatmaps were generated to illustrate patterns of expression 1092 of FABP7, FABP5, PMP2, SOX10, OLIG2 and ELAVL4. 


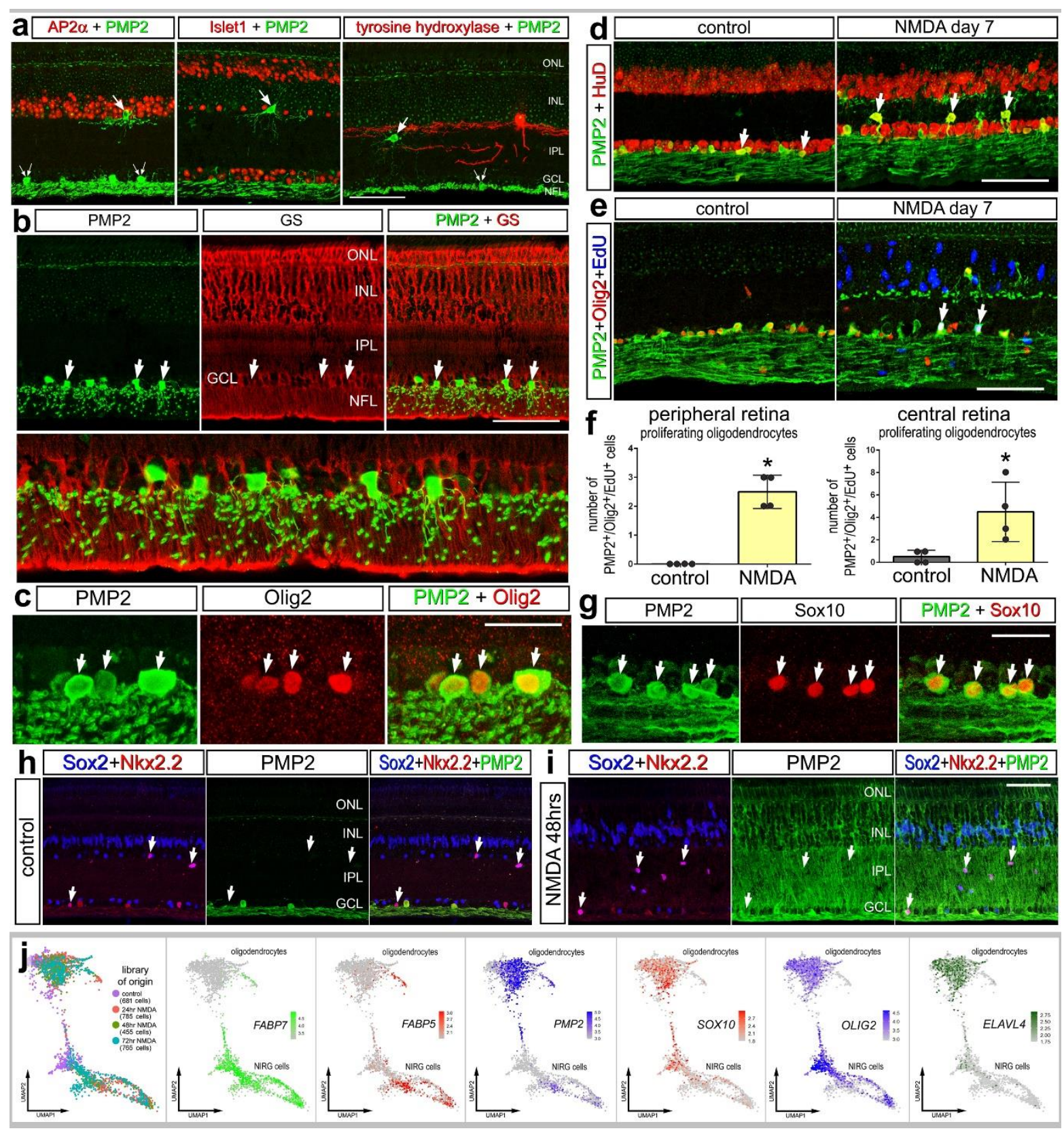


1102 Figure 4. Embryonic progenitors and MGPCs express different FABPs. MG were 1103 isolated from scRNA-seq libraries with significant numbers of MGPCs (retinas treated 1104 with NMDA and/or FGF2+insulin) and significant numbers of retinal progenitor cells (E5 1105 and E8 retinas). These cells were aggregated and ordered in UMAP plots (a-c). These 1106 unique clusters were probed for levels of expression of FABP5, FABP7, and PMP2 in 1107 UMAP heatmap plots (d-f), and quantified in violin plots $(\mathbf{g})$. Each dot in violin and 1108 scatter plots represents an individual cell. Significant changes $\left({ }^{*} p<0.1,{ }^{* *} p<0.0001\right.$, $\left.1109{ }^{* * *} \mathrm{p}<<0.0001\right)$ in levels were determined by using a Wilcox rank sum with Bonferroni 1110 correction. The eyes of post-hatch chicks were injected with EdU, insulin +FGF2 and/or 1111 FABP inhibitor (BMS309403) (h-k). Inhibition of FABPs had no effect upon the 1112 proliferation of $\mathrm{CMZ}$ progenitors (i-k). Sections of the far peripheral retina and $\mathrm{CMZ}$ 1113 were labeled for EdU-incorporation (red; $\mathbf{h}, \mathbf{j}, \mathbf{k}$, ) and antibodies to PMP2 (green; $\mathbf{h}$ ) or 1114 Sox2 (green; j,k). The histogram in i represents the mean ( \pm SD) and each dot 1115 represents one biological replicate retina. The calibration bar in k represents $50 \mu \mathrm{m}$ 1116 applies to $\mathbf{h}, \mathbf{j}$ and $\mathbf{k}$. Abbreviations: ONL - outer nuclear layer, INL - inner nuclear 1117 layer, IPL - inner plexiform layer, GCL - ganglion cell layer, CMZ - circumferential 1118 marginal zone. Significance of difference $\left({ }^{* *} \mathrm{p}<0.01\right)$ in mean numbers of EdU+ cells in 1119 the CMZ and peripheral INL was determined by using ANOVA followed by a post-hoc t1120 test with Bonferroni correction. 


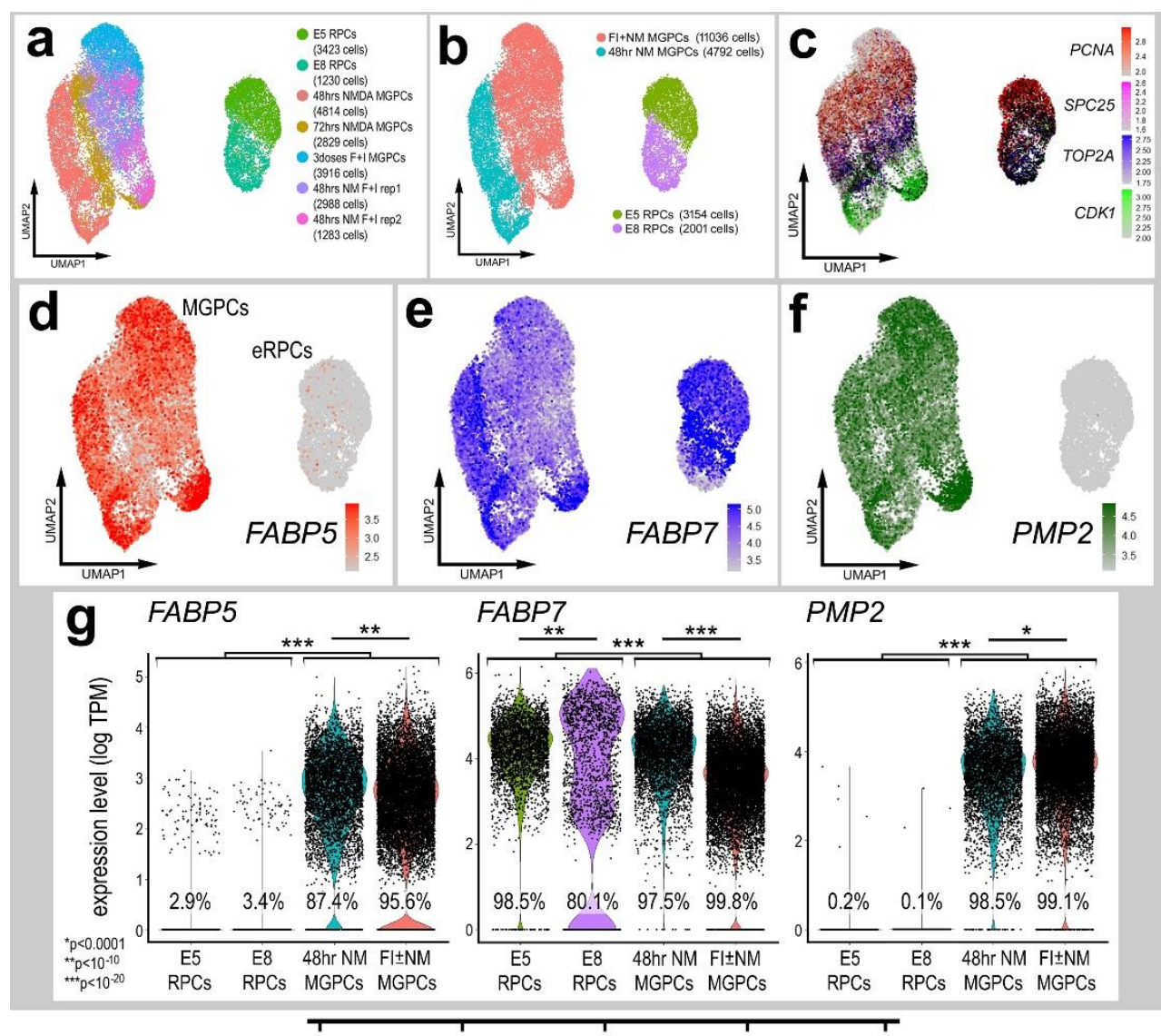

$\begin{array}{cccccc}\text { control } & E d U & E d U & E d U & E d U & \text { harvest } \\ \text { treated } & E d U+1+F & E d U+1+F & E d U+1+F & E d U & \end{array}$
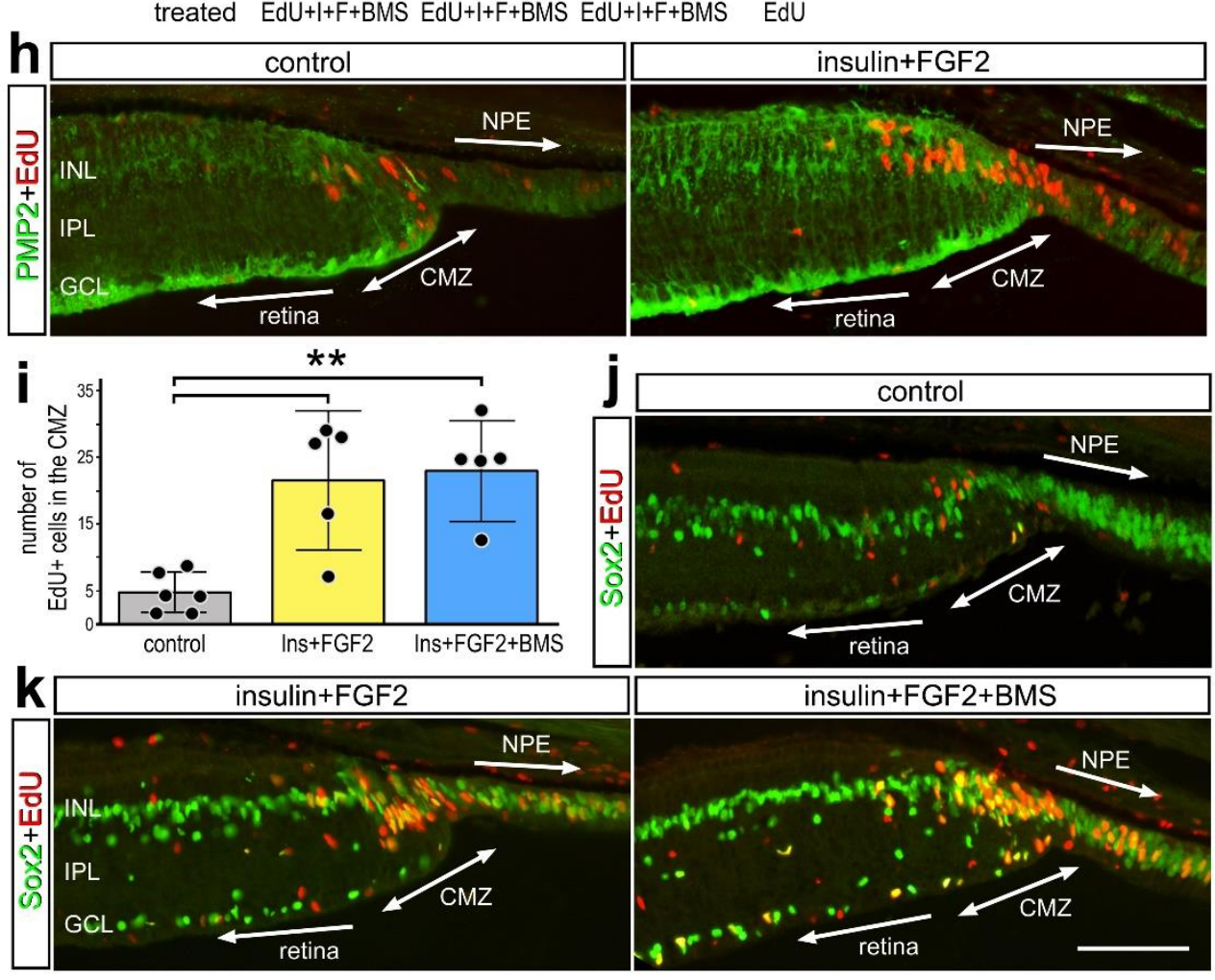
1124 Fig 5. FGF2 and insulin induce expression of PMP2 and FABP5 in MG. scRNA-seq

1125 were established for chick retinas treated with saline or two or three doses of FGF2 and

1126 insulin (a). UMAP ordering of cells revealed distinct clusters of retinal cell types (a).

1127 Resting MG and growth factor-treated MG formed distinct clusters based on patterns of 1128 expression including GLUL, RLBP1 and SLC1A3 (b). MGPCs were identified based on 1129 patterns of expression of NESTIN, CDK1 and TOP2A (b). UMAP heatmaps and violin

1130 plots were established to illustrate patterns and levels of expression for FABP5, FABP7

1131 and PMP2 (c and d). MG were re-embedded and ordered across pseudotime with a

1132 junction and trajectories populated by activated MG (mostly 2 doses of insulin and

1133 FGF2) or proliferating MGPGs branches (mostly 3 doses of insulin and FGF2) (e).

1134 Dimensional reduction of pseudotime to the X-axis placed resting MG (high $R L B P 1$ ) to

1135 the left and activated MG (black line) and MGPCs (red line; high CDK1) to the right (f).

1136 Levels of FABP5, FABP7 and PMP2 were plotted across pseudotime (f). 


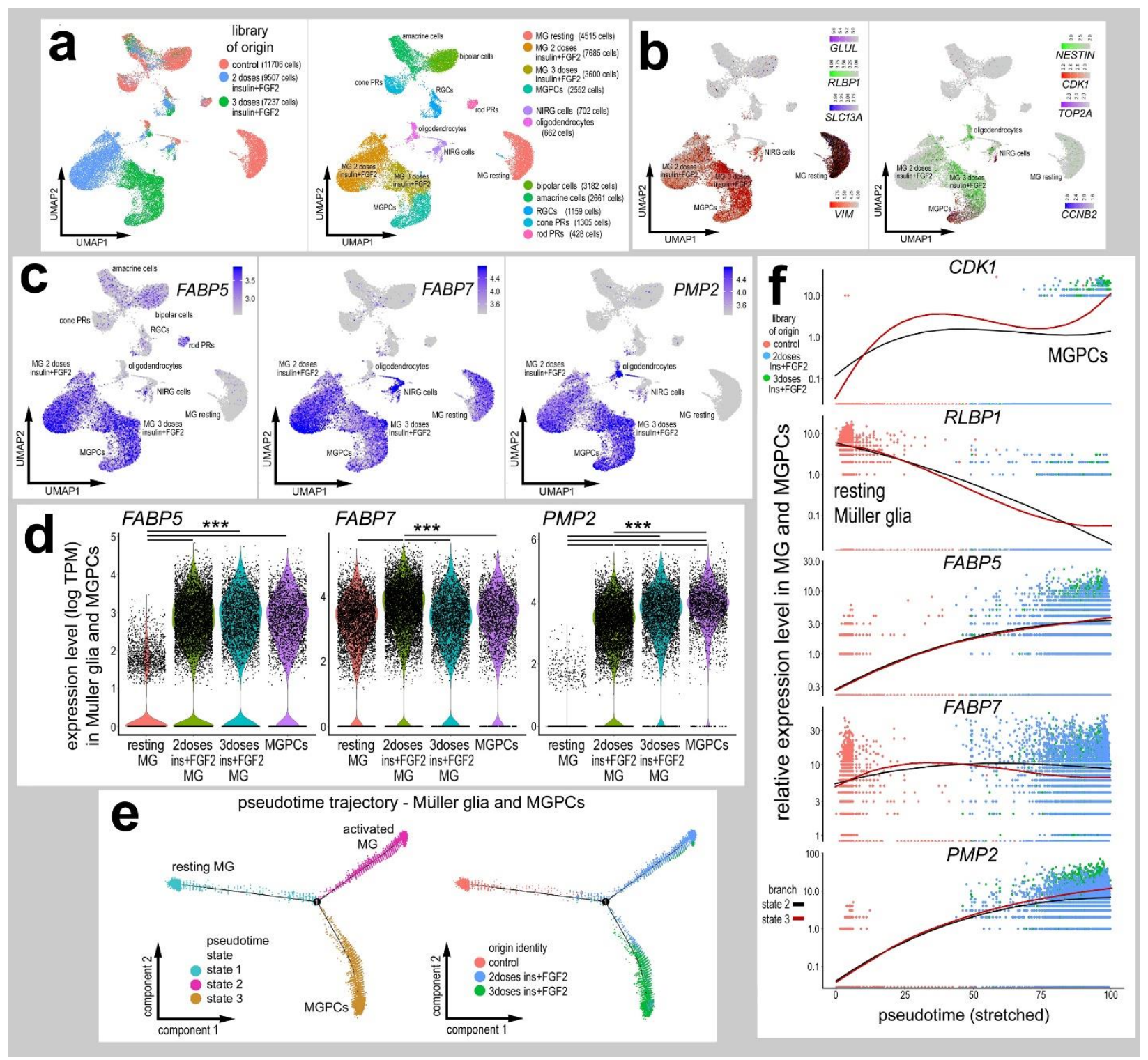


1149 Figure 6. Treatment of retinas with FGF2 stimulates MG to up-regulate PMP2 and 1150 proliferate, and this proliferation can be blocked by FABP-inhibitor. $(\mathbf{a}, \mathbf{b})$ Eyes were

1151 treated with 4 consecutive daily intraocular injections of FGF2, followed by 2

1152 consecutive daily injections of EdU, and retinas harvested $2 \mathrm{hrs}$ after the last injection.

1153 (c,d) Alternatively, eyes received 4 consecutive daily injections of FGF2 \pm BMS309403,

1154 followed by 2 consecutive daily injections of EdU, and eyes harvested $24 \mathrm{hrs}$ after the 1155 last injection. Sections of the retina were labeled for EdU (red; b) and antibodies to

1156 PMP2 (green; $\mathbf{a}, \mathbf{b}$ ) or Sox2 (blue in $\mathbf{b}$, green in $\mathbf{c}$ ). Arrows indicate MG nuclei labeled

1157 for EdU and Sox2. The histogram in $\mathbf{d}$ represents the mean $( \pm \mathrm{SD})$ and each dot

1158 represents one biological replicate retina. Significance $\left({ }^{*} \mathrm{p}<0.01\right)$ of difference was

1159 determined by using a Student's t-test. The calibration bar represents $50 \mu \mathrm{m}$. 

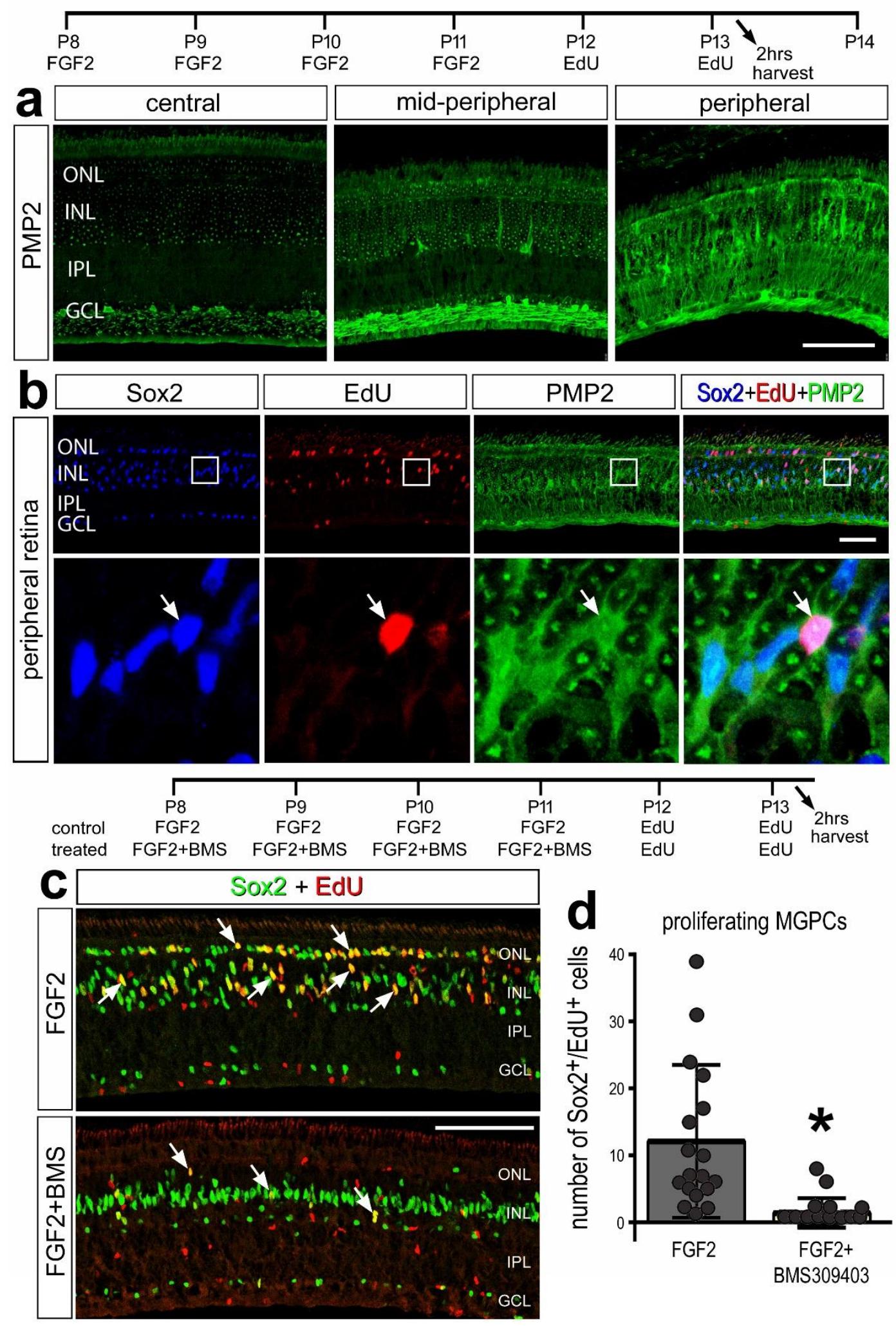
1165 Figure 7. Inhibition of FABP significantly impacts single cell transcriptomic

1166 profiles of MG. Retinas were treated with saline \pm BMS309403 or NMDA \pm

1167 BMS309403, and scRNA seq libraries were generated to analyze changes in MG gene

1168 expression. UMAP ordering of cells was established and MG were identified based on

1169 expression of genes associated with activated glia (d), proliferating MGPCs (e), and

1170 resting glia (f). Differentially expressed genes (DEGs) were identified for MG from

1171 retinas treated with saline vs BMS-saline, saline vs NMDA, and NMDA vs BMS-NMDA

1172 and plotted in a Venn diagram (c). Dot plots indicating the percentage of expressing MG

1173 (size) and expression levels (heatmap) for genes related to resting glia, secreted

1174 factors, glial transcription factors, inflammation, glial reactivity and proliferation $(\mathbf{g}, \mathbf{h})$. All

1175 genes displayed in the Dot plot have significantly different $(p<0.0001)$ expression levels

1176 in MG from retinas treated with saline vs saline-BMS $(\mathbf{g})$ or in $M G$ from retinas treated

1177 with NMDA vs NMDA-BMS (h). Gene Ontology (GO) terms for the enriched genes in

1178 the BMS treated and BMS+NMDA treated were compiled (ShinyGO) and grouped by

1179 biological process, cellular component and molecular function. GO enrichment analysis

1180 was performed. The significance of the function and the number of enriched genes are

1181 listed for each GO category. 


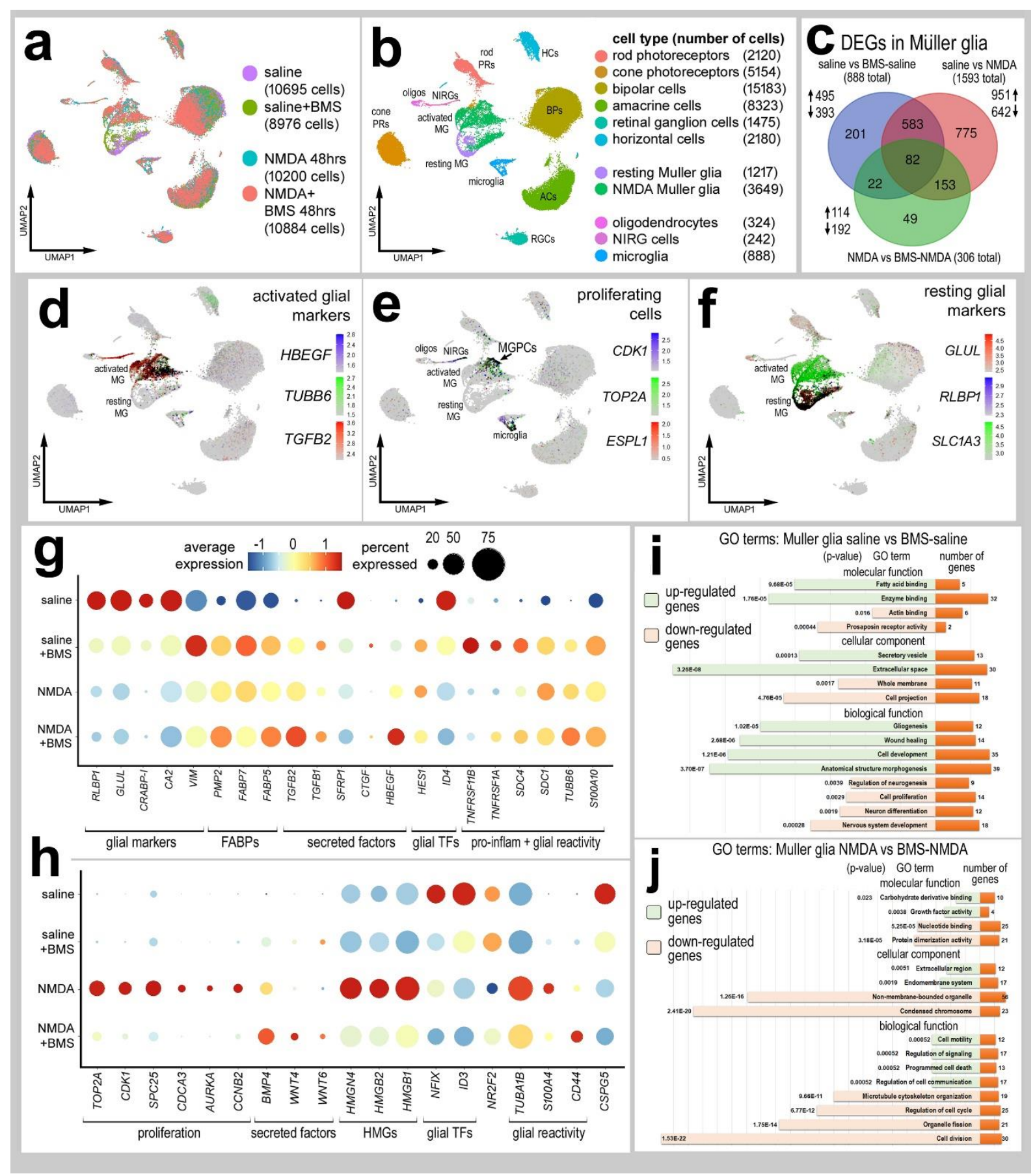


1190 Figure 8. Ligand-receptor (LR) interactions inferred from scRNA-seq data between

1191 microglia, NIRG cells, resting MG, activated MG and MGPCs. Retinal glia were isolated,

1192 re-embedded and ordered in UMAP plots to reveal distinct clusters of cells $(\mathbf{a}, \mathbf{b})$. The 1193 resting MG were comprised primarily of MG from saline-treated retinas, activated MG

1194 were comprised of cells from BMS-saline, NMDA and BMS-NMDA treated retinas, and

1195 MGPCs were primarily comprised of cells from NMDA-treated retinas (a-c). Glia from

1196 different treatment groups were analyzed using SingleCellSignalR to generate chord

1197 diagrams and illustrate potential autocrine and paracrine LR-interactions (d-g). LR-

1198 interactions were identified for glial cells for different treatment groups including saline

1199 (h,l), BMS-saline (i,m), NMDA (j,n) and BMS-NMDA (k,o). For each treatment group, 1200 the 40 most significant LR-interactions between microglia to resting MG (h,i), microglia 1201 to NIRG cells (I), microglia to activated MG (j,k,m), and activated MG to MGPCs (n-o) 1202 were identified and illustrated in chord plots and LRscore heat maps. Treatment-specific 1203 differences in glial LR-interactions in saline vs BMS-saline (p) and NMDA vs BMS-

1204 NMDA (q) are illustrated in Venn diagrams with some key interactions listed. 


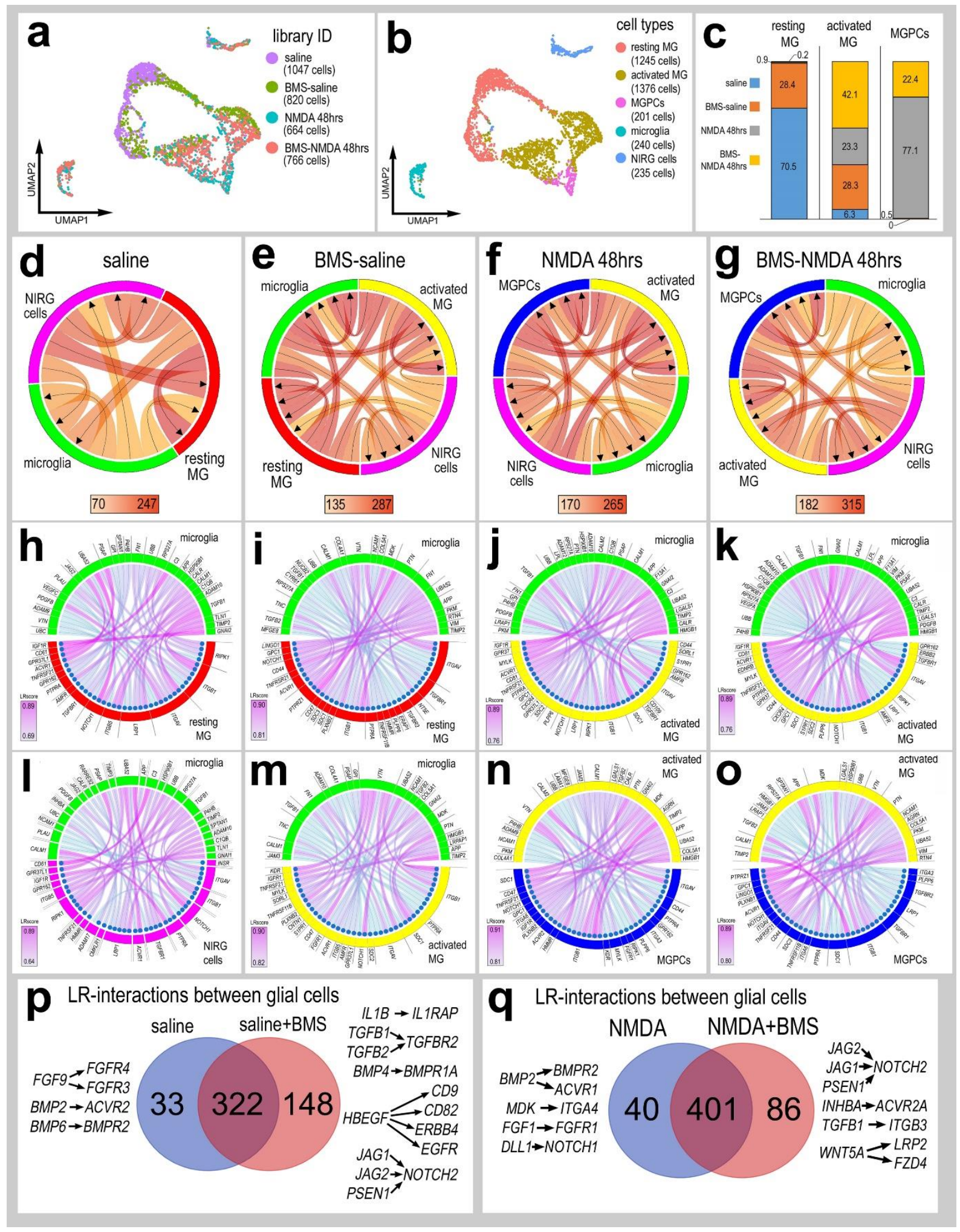


1210 Figure 9. (a-b) Inhibition of FABPs suppresses multiple cell signaling pathways in

1211 NMDA-damaged retinas. BMS309403 or vehicle were injected at P6 with NMDA,

1212 followed by injection of vehicle or BMS309403 at P7, and retinas were harvested 4

1213 hours after the last injection. (a) Retinal sections were labeled for fragmented DNA

1214 (TUNEL; red) or antibodies to pS6 (green) and Sox2 (red), pStat3 (green), or

1215 pSmad1/5/8 (green). The histograms in $\mathbf{b}$ illustrate numbers of dying cells or

1216 immunofluorescence intensity sum for pS6, pStat3, and pSmad1/5/8. (c-d) Inhibition of

1217 FABPs in undamaged retinas up-regulates vimentin and PMP2 in MG, and suppresses

1218 the formation of MGPCs when damage is induced. Sections of the retina were labeled

1219 with antibodies to vimentin or PMP2 (c). The histogram in $\mathbf{d}$ illustrates levels of

1220 immunofluorescence intensity sum for vimentin and PMP2. (e-f) BMS309403 was

1221 injected at P6 and P7, NMDA was injected at P8, EdU was injected at P9 and P10, and

1222 retinas were harvested at P11. Retinal sections were labeled for EdU and antibodies to

1223 Sox2 (e). Arrows indicate the nuclei of MG labeled for Sox2 and EdU in the INL, hollow

1224 arrow-heads indicate the nuclei of NIRG cells labeled for Sox2 and EdU in the IPL, and

1225 small double-arrows indicate putative proliferating microglia labeled for EdU alone. The

1226 calibration bars in $\mathbf{a}, \mathbf{c}$ and $\mathbf{e}$ represents $50 \mu \mathrm{m}$. The histogram in $\mathbf{f}$ illustrates numbers

1227 of Sox2+/EdU+ MGPCs. Abbreviations: ONL - outer nuclear layer, INL - inner nuclear

1228 layer, IPL - inner plexiform layer, GCL - ganglion cell layer. The histograms illustrated

1229 the mean $( \pm S D)$ and each dot represents one biological replicate retina. Significance

$1230 \quad\left({ }^{*} \mathrm{p}<0.05,{ }^{* *} \mathrm{p}<0.01,{ }^{* * *} \mathrm{p}<0.001\right)$ of difference was determined by using a Student's t-

1231 test. 


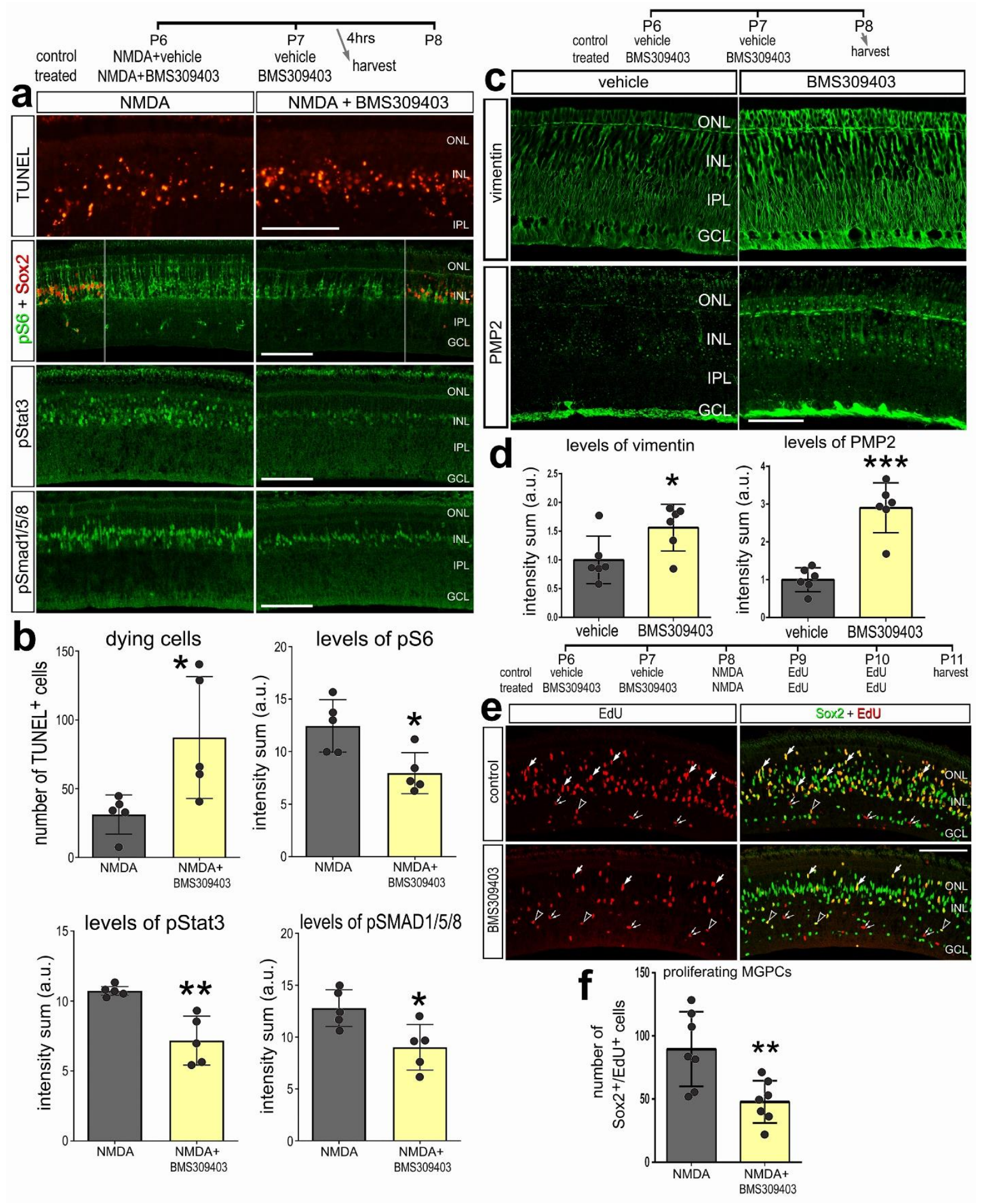


1235 Figure 10. FABP inhibition reduces microglia proliferation. Inhibition of FABPs with

1236 BMS309403 suppressed the accumulation and proliferation of microglia (a,b). Sections

1237 of the retina were labeled for DRAQ5 (red) and CD45 (green; a). The calibration bar in a 1238 represents $50 \mu \mathrm{m}$. Abbreviations: ONL - outer nuclear layer, INL - inner nuclear layer, $1239 \mathrm{IPL}$ - inner plexiform layer, GCL - ganglion cell layer. The histogram in $\mathbf{b}$ represents the 1240 mean ( \pm SD) number of CD45+/EdU+ cells and each dot represents one biological 1241 replicate. Significance of difference $\left({ }^{*} \mathrm{p}<0.01\right)$ was determined using a two-way paired t1242 test. scRNA-seq libraries established from retinas treated with saline \pm BMS or NMDA \pm 1243 BMS and microglia were isolated and analyzed for changes in genes expression. The 1244 microglia were clustered by UMAP and analyzed for differentially expressed genes 1245 (DEGs) which are illustrated in a Venn diagram (g). Dot plots indicating the percentage 1246 of expressing microglia (size) and expression level (heatmap) were generated for 1247 classes of genes including those important for proliferation, transcriptional regulation, 1248 cell signaling and inflammatory signaling (e). All genes displayed in the dot plot have 1249 significantly different $(p<0.0001)$ expression levels in microglia from retinas treated with 1250 saline vs saline-BMS. Gene Ontology terms for the enriched genes in the BMS treated 1251 and BMS+NMDA treated were compiled (ShinyGO) (g,h,i). GO was performed for 1252 significantly up-regulated genes (green) and the number of enriched genes in each GO 1253 category (orange) are displayed. There were insufficient numbers of down-regulated 1254 genes and insufficient DEGs identified for NMDA vs NMDA+BMS to perform GO 1255 enrichment analyses. Violin plots of FABP5, FABP7, PMP2 and FASN illustrate the 1256 percentage of expressing cells and significant changes in levels of expression between 

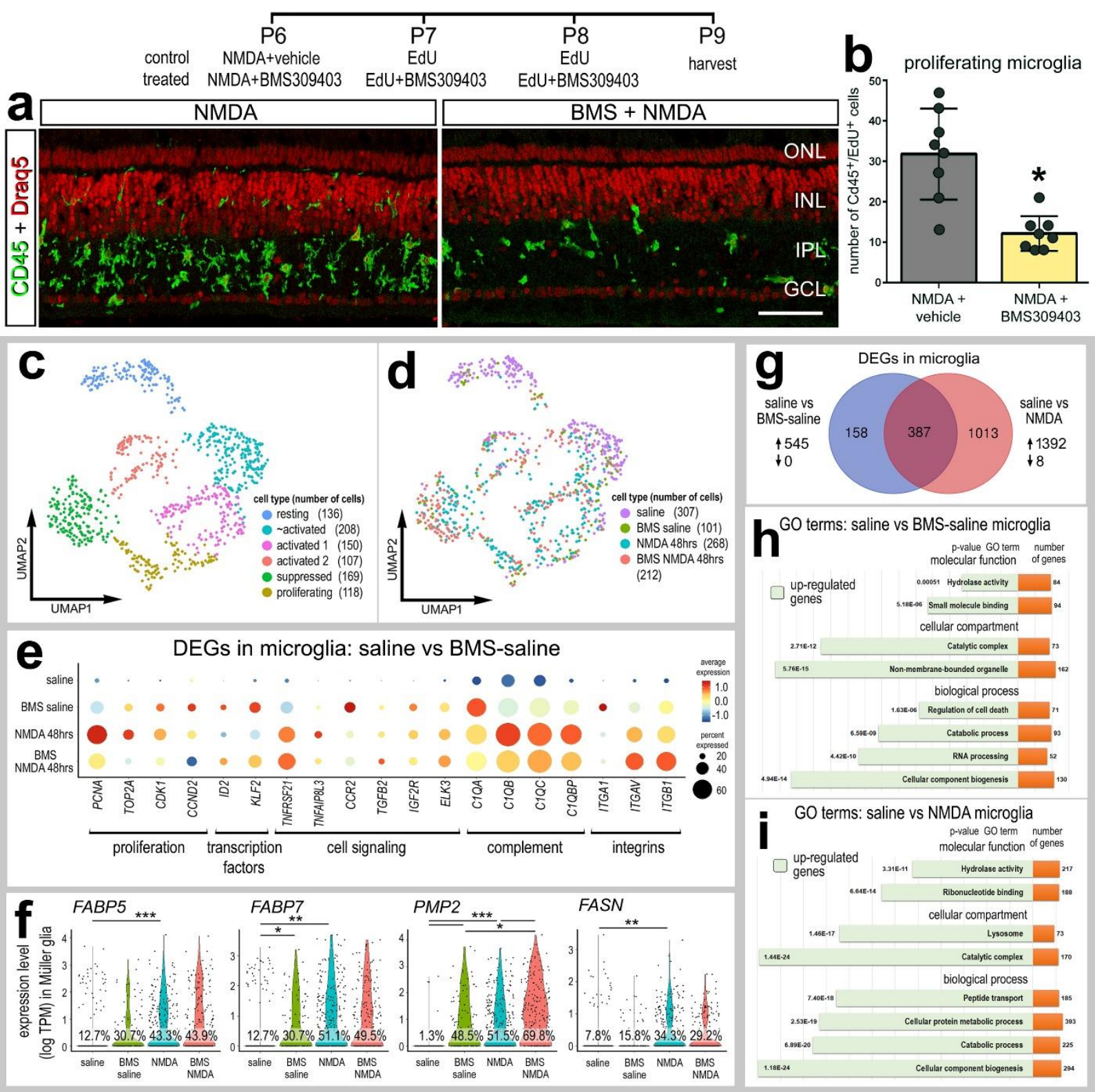
Figure 11. Fatty acids synthase inhibitors significantly reduce MGPC formation.

1267 scRNA-seq libraries of the NMDA damaged retinas were probed Fatty acids synthase

1268 (FASN). UMAP heatmap plot in a illustrate FASN expression across different cells types

1269 from retinas treated with saline or NMDA at 24, 48 or 72 hrs after treatment. Expression

1270 levels and percentage expressing cells in MG are illustrated in violin plots $(\mathbf{b}, \mathbf{d})$. The

1271 UMAP plot in $\mathbf{c}$ illustrates discrete clustering of MG from retinas treated with saline or

1272 NMDA at 3, 12 or 48 hrs after treatment. FASN inhibitors C75 and G28UCM were

1273 applied either with or following NMDA. Retinal sections were labeled for EdU (red) and

1274 Sox2 (green; e), TUNEL (h), or DRAQ5 (magenta), EdU (red) and CD45 (green; k,l).

1275 Arrows indicate nuclei of MG, small double-arrows nuceli of NIRG cells and hollow

1276 arrow-heads indicate nuclei of microglia. The histograms $\mathbf{f}, \mathbf{g}, \mathbf{i}, \mathbf{j}$ and $m-p$ represents

1277 the mean $( \pm$ SD) and each dot represents one biological replicate retina. The calibration

1278 bar in c represents $50 \mu \mathrm{m}$. Abbreviations: ONL - outer nuclear layer, INL - inner

1279 nuclear layer, IPL - inner plexiform layer, GCL - ganglion cell layer. Significant

1280 changes $\left({ }^{*} \mathrm{p}<0.01,{ }^{* *} \mathrm{p}<0.001,{ }^{* * *} \mathrm{p}<0.0001\right)$ in expression were determined by using a

1281 Wilcox rank sum with Bonferroni correction. 

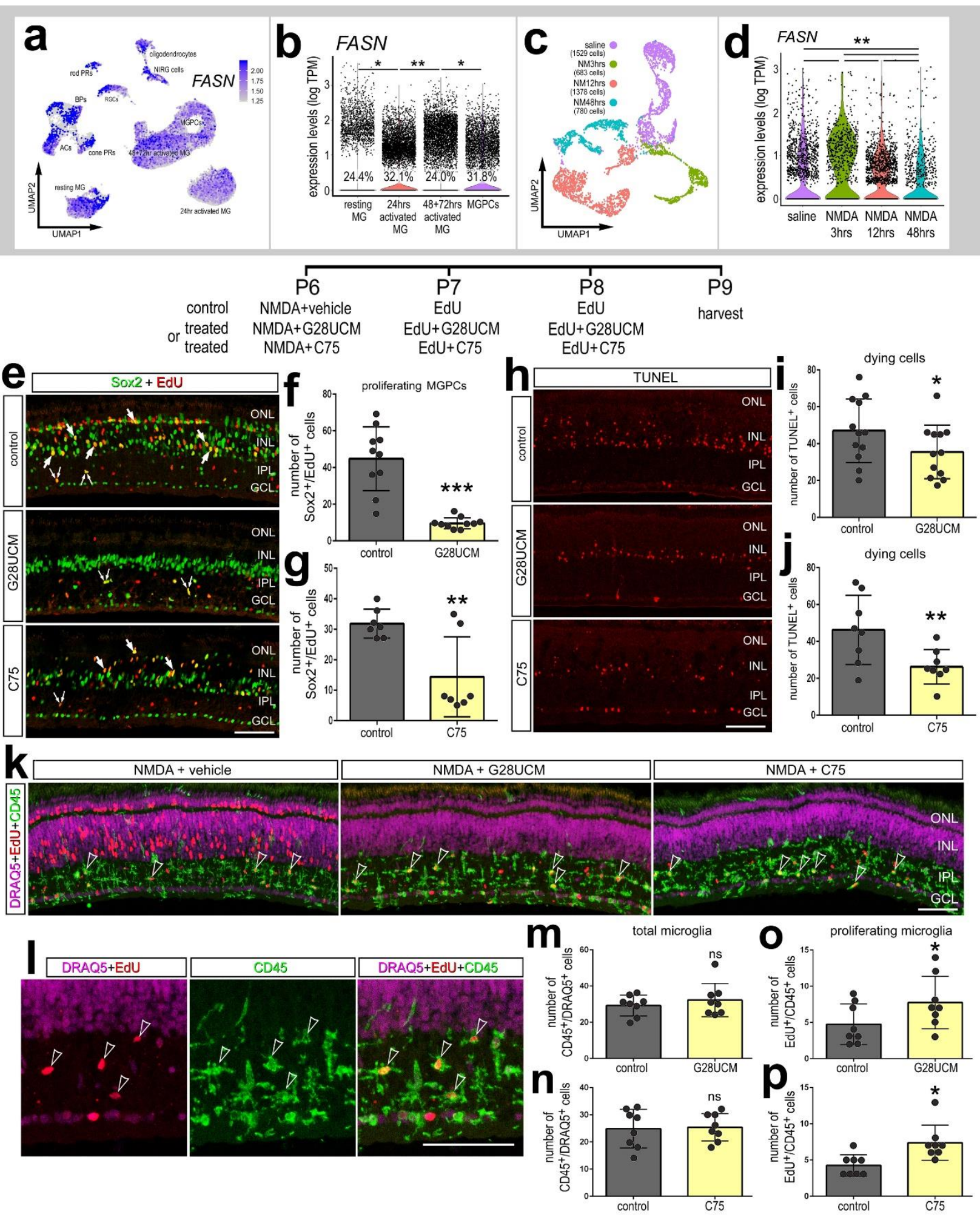
1288 Figure 12. Inhibition of FASN suppresses cell signaling in MG. Retinas were obtained

1289 from eye injected with NMDA \pm G28UCM (FASN inhibitor) at P7, vehicle \pm G28UCM

1290 and harvested 4 hours after the last injection. Retinal sections were labeled for Sox2

1291 (red) and pS6 (green), pStat3 (green), pSmad1/5/8 (green), and fragmented DNA in the

1292 nuclei of dying cells (a). Arrows indicate the nuclei of MG. The calibration bar in a

1293 represents $50 \mu \mathrm{m}$. Abbreviations: ONL - outer nuclear layer, INL - inner nuclear layer,

$1294 \mathrm{IPL}-$ inner plexiform layer, $\mathrm{GCL}$ - ganglion cell layer. The histograms represent the

1295 mean $( \pm$ SD) fluorescence intensity sum (a.u.; b) or mean number of TUNEL+ cells (c)

1296 and each dot represents one biological replicate retina. Significance ( $p$-values) of

1297 difference was determined by using a two-tailed paired Student's t-test. 


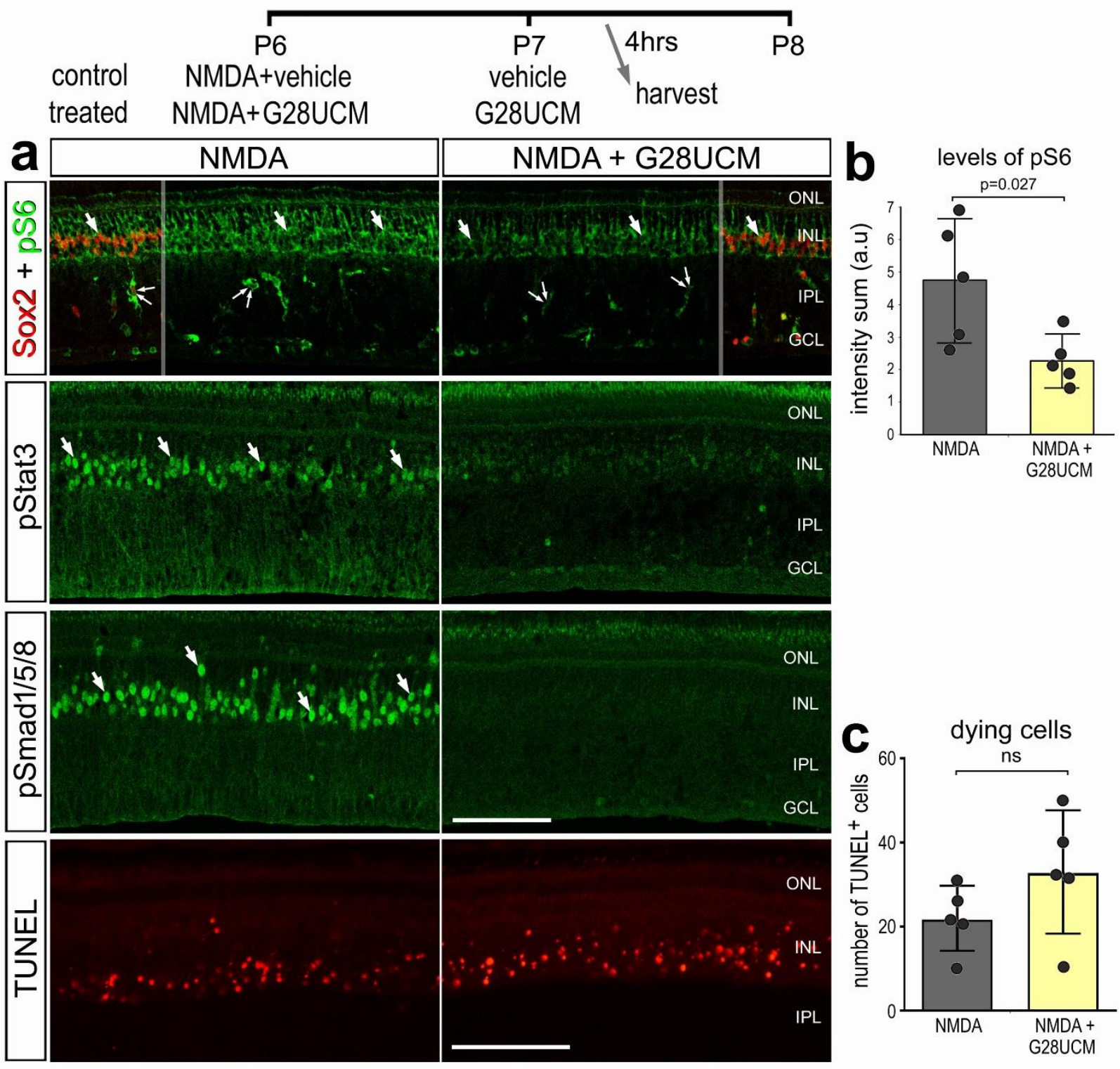


1306 Supplemental Figure 1. scRNA-seq was used to interrogate expression of FABPs in 1307 MG at timepoints soon after damage. Retinas were harvested at 3, 12 or 48 hrs after

1308 treatment with NMDA. UMAP plots illustrate the distribution of different libraries (saline, 1309 4hrs, 12hrs and 48hrs after NMDA) (a). UMAP ordering of cells revealed 7 different

1310 clusters of cells (b). Resting MG were identified based on elevated levels of expression

1311 for CA2, RLBP1, GLUL and CSPG5, whereas activated MG down-regulated these

1312 markers (c). MGPCs were identified based on expression of proliferation markers

1313 including SPC25, CDCA3, TOP2A and CDK1 (d). UMAP heatmap plots illustrate

1314 patterns of expression of $F A B P 5(\mathbf{e}), F A B P 7(\mathbf{f})$ and PMP2 (g). Expression levels (log

1315 TPM) and the percentage of expressing MG are illustrated in violin plots (h).

1316 Significance $\left({ }^{*} \mathrm{p}<0.01,{ }^{* *} \mathrm{p}<0.0001\right)$ of difference in expression was determined by

1317 using a Wilcox rank sum with Bonferroni correction. 


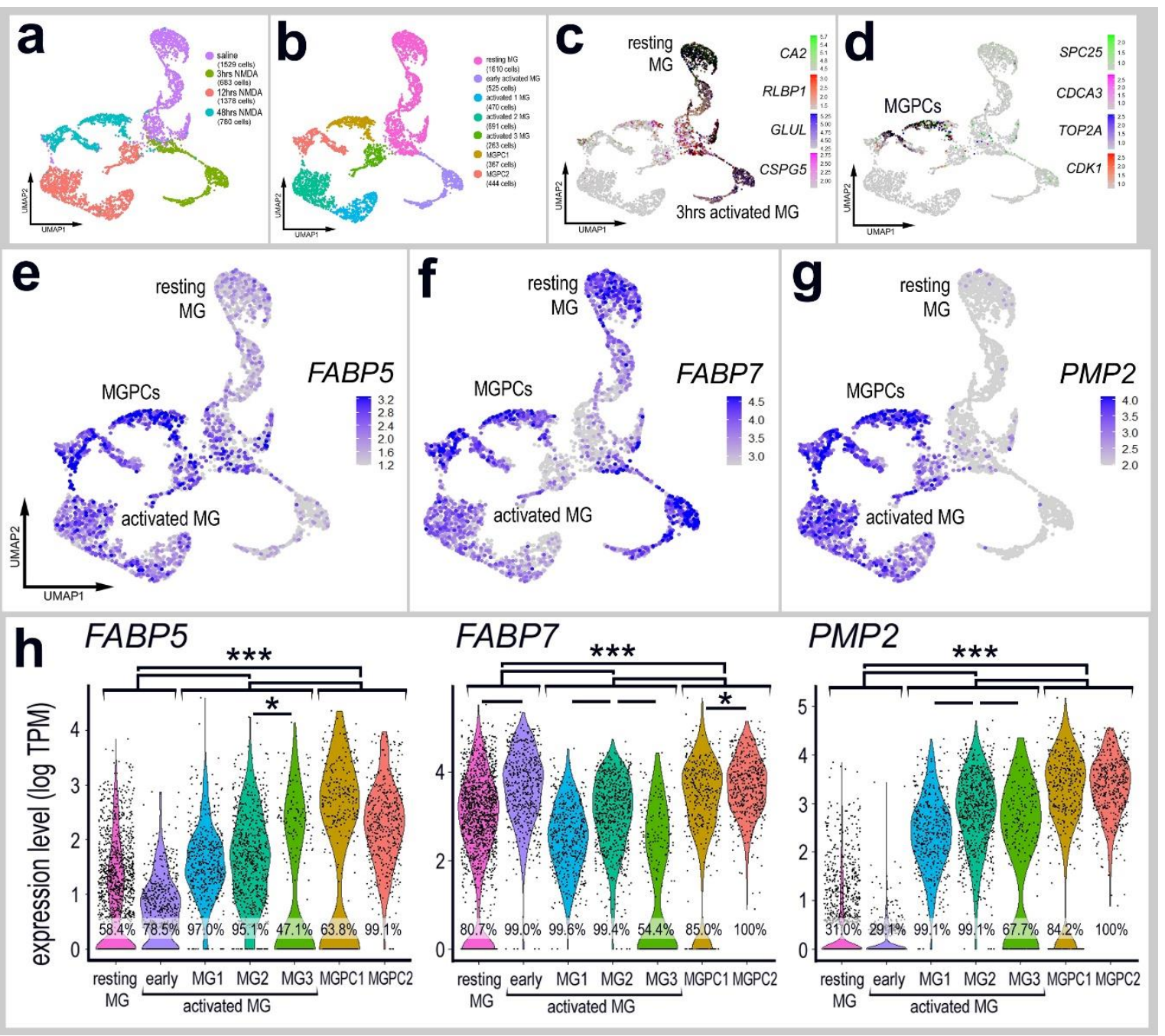


1330 Supplemental Figure 2. Comparison of FABP and FASN expression levels in MG and

1331 MGPCs from different treatment conditions. scRNA-seq was used to identify patterns of

1332 expression of FABPs and FASN in MG and MGPCs at different time points after NMDA

1333 damage and/or FGF + insulin growth factor treatment. Nine different libraries were

1334 aggregated for a total of more than 55,000 MG and MGPCs (a-c). MGPCs were

1335 identified based on high-levels of expression of CDK1, TOP2A, PCNA and SPC25 (e),

1336 and were a mix of cells from 48hrs NMDA+FGF2+insulin, 48hrs NMDA, 72hrs NMDA

1337 and 3 doses of insulin+FGF2 (b). Resting MG were identified based on expression of

1338 GLUL, RLBP1, SLC1A3 and VIM (c,d). Each dot represents one cell and black dots

1339 indicate cells with 2 or more genes expressed. The expression of FABP5, FABP7,

1340 PMP2 and FASN is illustrated UMAP and violin plots with population percentages and

1341 statistical comparisons $(\mathbf{f}-\mathbf{h})$. Significance of difference $\left({ }^{* * *} p<10-20\right)$ in expression levels

1342 (log TPM) were determined by using a Wilcox rank sum with Bonferoni correction.

1343 Abbreviations: ONL - outer nuclear layer, INL -inner nuclear layer, IPL -inner plexiform

1344 layer, GCL -ganglion cell layer. 


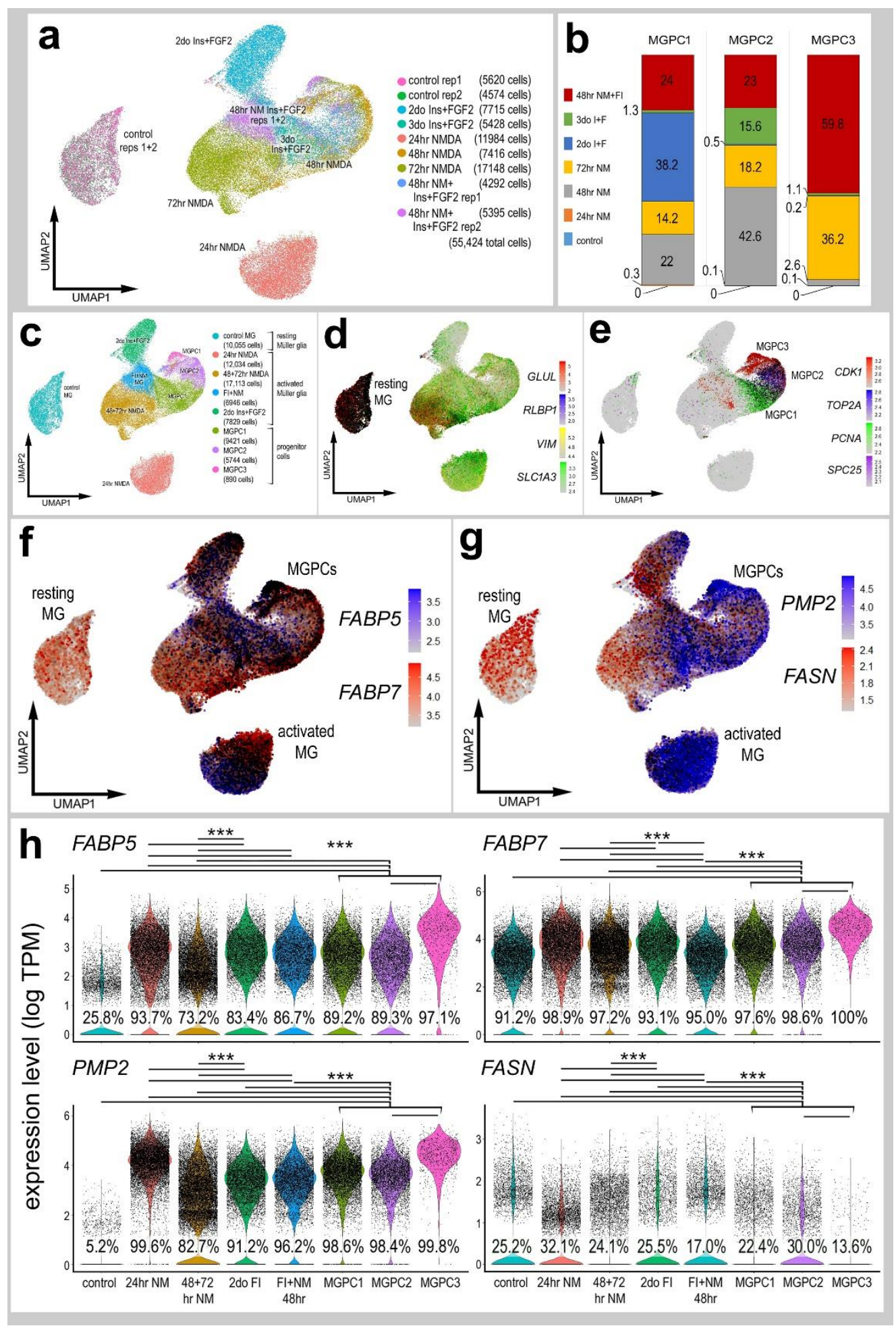


1351 Supplemental Figure 3. scRNA seq libraries were generated to analyze changes in

1352 MG gene expression. MG were identified based on expression of genes associated with

1353 resting glia, activated glia, and proliferating MGPCs. UMAP ordering of MG revealed 2

1354 clusters of resting MG, 2 clusters of activated MG, and one cluster of MGPCs (a).

1355 Resting MG clusters were predominantly occupied by MG from saline-treated retinas,

1356 activated MG clusters were occupied by cells treated with saline-BMS, NMDA alone and

1357 NMDA-BMS, and the MGPC cluster was predominantly occupied by cells from NMDA-

1358 treated retinas (b,c). Resting MG were identified based on expression of markers such

1359 as CA2, GLUL and RLBP1 (d). MGPCs were identified based on expression of

1360 proliferation markers such as CDK1, SPC25 and TOP2A (e). Activated MG were

1361 identified based on expression of markers such as CD44, MANF and TGFB2 (f).

1362 Differentially expressed genes (DEGs) were identified for MG from retinas treated with

1363 saline vs BMS-saline, saline vs NMDA, and NMDA vs BMS-NMDA and plotted in a

1364 Venn diagram (i). The Dot plot plots indicating the percentage of expressing MG (size)

1365 and expression levels (heatmap) for genes related to resting glia, secreted factors, glial

1366 transcription factors, inflammation, glial reactivity and proliferation $(\mathbf{g}, \mathbf{h})$. All genes

1367 displayed in the Dot plot have significantly different $(p<0.0001)$ expression levels in MG

1368 from retinas treated with saline vs saline-BMS $(\mathbf{g})$. Gene Ontology (GO) terms for the

1369 enriched genes in the BMS treated and BMS+NMDA treated were compiled (ShinyGO)

1370 and grouped by biological process, cellular component and molecular function. GO

1371 enrichment analysis was performed for up-regulated DEGs (light green) and down-

1372 regulated DEGs (light orange). The significance of the GO category and the number of 1373 enriched genes grouped into each function (orange) are displayed. 
bioRxiv preprint doi: https://doi.org/10.1101/2021.08.19.456977; this version posted August 19, 2021. The copyright holder for this preprint (which was not certified by peer review) is the author/funder. All rights reserved. No reuse allowed without permission.

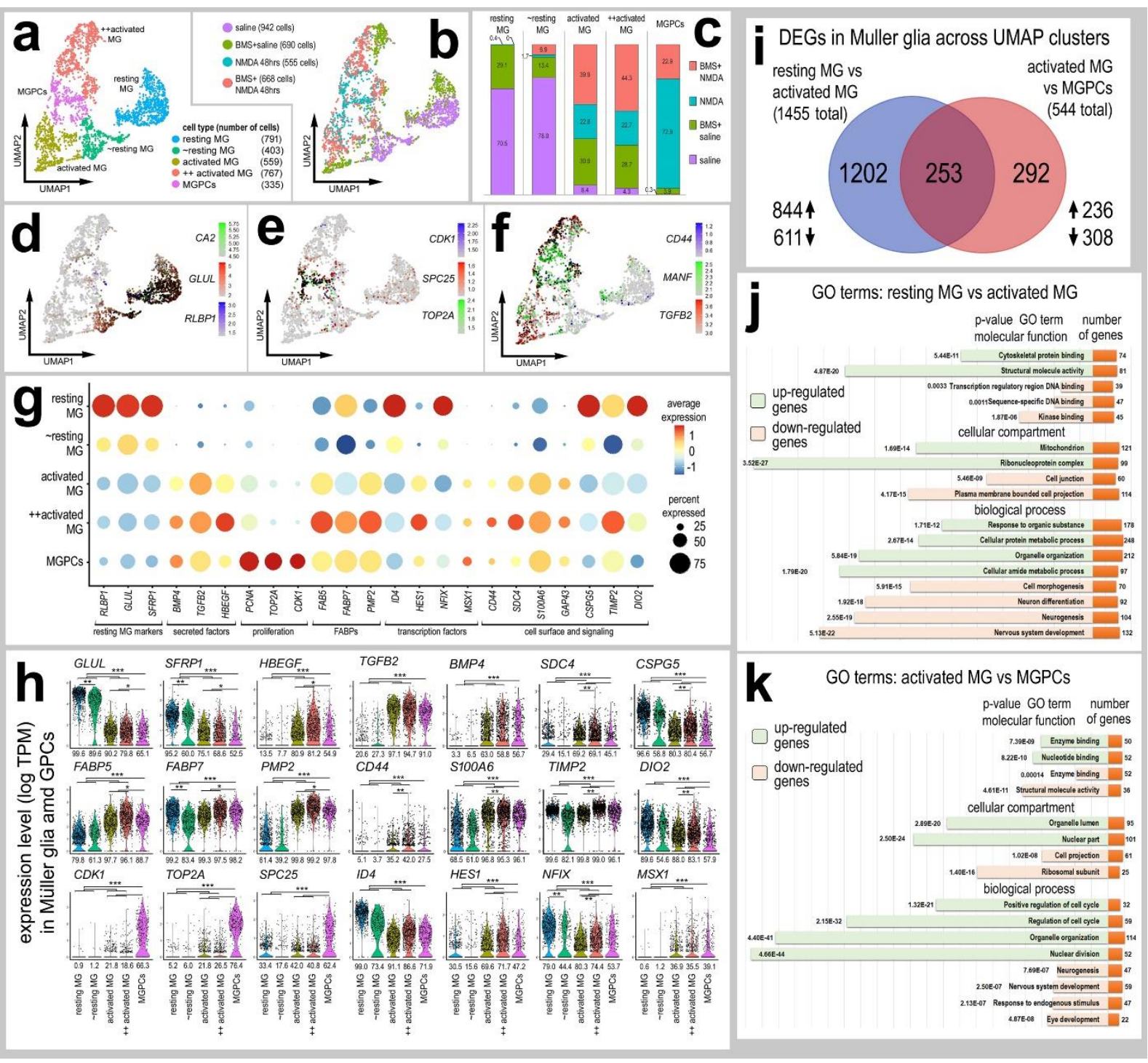

